\title{
PRIMEIRA LISTA DO MATERIAL-TIPO DE COLEOPTERA DA COLEÇÃO DE ENTOMOLOGIA PE. J.S. MOURE, DO DEPARTAMENTO DE ZOOLOGIA DA UNIVERSIDADE FEDERAL DO PARANÁ ${ }^{1}$
}

\author{
Renato C. Marinoni ${ }^{2}$ \\ Lúcia M. de Almeida ${ }^{2}$ \\ Dilma S. Napp ${ }^{2}$ \\ Germano H. Rosado-Neto ${ }^{2}$
}

\begin{abstract}
A FIRST LIST OF COLEOPTERA TYPE-MATERIAL OF THE ENTOMOLOGICAL COLLECTION PE. J.S. MOURE OF THE "DEPARTAMENTO DEZOOLOGIA DA UNIVERSIDADE FEDERALDO PARANÁ". A list of 1,302 type-specimens of the Families Belidae, Bruchidae, Buprestidae, Bostrichidae, Carabidae, Cerambycidae, Chrysomelidae, Coccinellidae, Curculionidae, Erotylidae, Gyrinidae, Scarabaeidae, Scolytidae and Staphylinidae is presented. From the total, 92 are holotypes; 1,059 paratypes; 19 alotypes; 204 types; and 10 cotypes.

KEY WORDS. Insecta, Coleoptera, type-material
\end{abstract}

O Departamento de Zoologia da Universidade Federal do Paraná, desde os seus primórdios, teve uma linha de pesquisa fundamentada em Sistemática de Insetos, através do trabalho do seu Professor Catedrático, Padre Jesus Santiago Moure.

Foi a partir de 1956 que a coleção entomológica passou a ser formada, pois, até então, o material coletado era depositado junto à coleção do antigo Museu Paranaense. Até 1962, ela teve seu maior desenvolvimento no grupo de Hymenoptera-Apoidea, em razão dos trabalhos do Padre Moure e das Professoras Danúncia Urban e Bernadete Lucas de Oliveira. Os demais grupos de insetos também estavam representados, mas em razão, principalmente, da compra da coleção F. Justus Junior, em fins da década de 50, contendo cerca de 8.000 exemplares. A partir de 1962, a coleção passou a sofrer contínuos acréscimos pelos trabalhos de um coletor (Claudionor Elias) atuando inicialmente na área do sul de Minas Gerais (Passos, Araxá, Ibiá, etc.) e, posteriormente, no Estado do Espírito Santo (Linhares, Baixo Guandu, Santa Teresa, Conceição da Barra, etc.). Este alento deveu-se, principalmente, às contribuições oriundas do então Conselho de Pesquisas da Universidade do Paraná, do Conselho Nacional de Desenvolvimento Científico e Tecnológico

1) Contribuição número 746 do Departamento de Zoologia, Universidade Federal do Paraná.

2) Departamento de Zoologia, Universidade Federal do Paraná, Caixa Postal 19020, 81531-970 Curitiba, Paraná, Brasil. Pesquisadores do CNPq. 
(CNPq) e da Fundação Rockefeller. A partir de 1963, novos professores ingressaram no Departamento e com o crescimento das atividades de pesquisas taxonômicas em Coleoptera (Cerambycidae), Lepidoptera (Hesperiidae) e Homoptera (Membracidae), também cresceu a representação destas Ordens na coleção.

Com o decorrer dos anos, em função do aumento do quadro de professores e do envolvimento de alunos pós-graduandos, mais grupos de insetos passaram a ser estudados, com conseqüente aumento da coleção, destacandose: Curculionidae, Chrysomelidae, Coccinellidae e Bruchidae (Coleoptera); Nymphalidae (Lepidoptera); Ichneumonidae (Hymenoptera); Isoptera; Muscidae e Calliphoridae (Diptera), Aphididae e Cicadellidae (Homoptera).

Hoje, a Coleção de Entomologia Pe. J.S. Moure do Departamento de Zoologia da Universidade Federal do Paraná (DZUP), conta com mais de 5.000 .000 exemplares (vias seca e úmida), resultantes da coleta por parte de membros do Departamento; da atividade do Centro de Identificação de Insetos Fitófagos (principalmente através do Projeto de Levantamento da Fauna Entomológica do Estado do Paraná PROFAUPAR), e pela aquisição de importantes coleçóes particulares, como as de Paulo Gagarin, Romualdo Ferreira d'Almeida, Heinz Ebert, Fritz Plaumann, Anton Maller, Moacir Alvarenga e Dmytro Zajciw.

Foi extremamente significativa para o estudo da Ordem Coleoptera, a aquisição das quatro últimas coleções, mesmo considerando o fato de terem sido compradas apenas parte de cada uma delas. A coleção de Fritz Plaumann, com cerca de 10.000 exemplares, contava com grande número de exemplarestipos, principalmente das famílias Scolytidae e Gyrinidae. A coleção de Moacir Alvarenga, com aproximadamente 25.000 exemplares, trouxe notável acréscimo à família Erotylidae, apresentando além de holótipos e parátipos, uma grande quantidade de homeótipos. A coleção Anton Maller, apesar de não contar com material-tipo, enriqueceu sobremaneira a coleção, pela maior representatividade que trouxe às familias Scarabaeidae, Chrysomelidae, Cerambycidae e Curculionidae. E a coleção Dmitro Zajciw incorporou à coleção de Cerambycidae alguns exemplares-tipos. A coleção de Coleoptera também cresceu em decorrência da gentileza de doações e permutas feitas pelo Dr. Carlos Alberto Campos Seabra; pelo Museu de Zoologia da Universidade de São Paulo (Dr. Ubirajara R. Martins); pelo Museu Nacional, da Universidade Federal do Rio de Janeiro (Dr. Miguel A. Monné).

A lista de material-tipo adiante arrolada é resultado de um levantamento que procura organizar e atualizar as informações taxonômicas da Coleção de Entomologia Pe. J.S. Moure.

Esta lista é caracterizada pelos seguintes elementos: 1) contém apenas o material-tipo da Ordem Coleoptera; 2) as espécies estão relacionadas por ordem alfabética de Família e nestas por ordem alfabética de Gênero; 3) a citação do material-tipo consta de: a) nome original, binário ou trinário, com que a espécie ou subespécie foi descrita; b) nome do autor e data da publicação; 
c) publicação, com número do volume e página(s), onde se encontra a descrição;

d) categoria do tipo; e) sexo do exemplar ( $M=$ macho, $F=$ fêmea), quando possível, ou indicado pelo autor; f) número de exemplares; g) localidade, com o nome entre parênteses, quando existente, indicando o ponto de coleta dentro de uma região geográfica política maior (município, por exemplo); seguindo-se o nome do Estado, Província ou Departamento, mais o do País; h) data da coleta; i) coletor; j) indicação da coleção a que pertencia o exemplar quando da descrição da espécie.

Foi incluido na lista somente o material do qual foi possível obter a citação bibliográfica de descrição da espécie ou subespécie. Há, ainda, cerca de uma centena de exemplares, rotulados como material-tipo, que não tivemos condições de confirmar a publicação das descrições. Este material provém, principalmente, das coleções Plaumann e Alvarenga.

As abreviaturas constantes da lista indicam os nomes dos Estados, Províncias ou Departamentos, e respectivos Países:

AR-ARGENTINA, BA-Buenos Aires; CH-Chaco; FO-Formosa; MIMisiones; SA-Salta; TU-Tucuman.

BO-BOLIVIA, CO-Cochabamba; SC-Santa Cruz.

BR-BRASIL, AC-Acre; AM-Amazonas; AP-Amapá; BA-Bahia; ES-Espírito Santo; GO-Goiás; MG-Minas Gerais; MS-Mato Grosso do Sul; MT-Mato Grosso; PA-Pará; PB-Paraíba; PE-Pernambuco; PR-Paraná; RJ_ Rio de Janeiro; RN-Rio Grande do Norte; RO-Rondônia; RS-Rio Grande do Sul; SC-Santa Catarina; SP-São Paulo.

CR-COSTA RICA, CA-Cartago; GU-Guanacaste; Punta Arenas.

EQ-EQUADOR, BO-Bolivar; IM-Imbabura.

ES-EL SALVADOR, LI-La Libertad.

HO-HONDURAS, CO-Cortés.

ME-MÉXICO, CH-Chiapas; QR-Quintana Roo; SI-Sinaloa; VC-Vera Cruz.

PA-PANAMÁ, PA-Panamá; ZC-Zona do Canal.

PE-PERU, AP-Apurimac; JU-Junin.

TB-TRINIDAD-TOBAGO, TR-Trinidad.

UR-URUGUAI, LC-La Colonia.

VE-VENEZUELA, AP-Apure; GU-Guarico; LA-Lara; ME-Merida; ZU-Zulia.

\section{BELIDAE}

Dicordylus serranus Vanin, 1976, Arq.Zool., S.Paulo, 28:14-15.

PARÁTIPO, F, Mafra, SC, BR, 02/65, A.Maller.

Homalocerus longirostris Vanin, 1976, Arq.Zool., S.Paulo, 28:28-29.

HOLÓTIPO, M, S.José do Barreiro (Serra da Bocaina), SP, BR, 11/68, Alvarenga \& Seabra. 


\section{BOSTRICHIDAE}

Ctenobostrychus alverneri Reichardt, 1962, Papéis Avulsos Dep. Zool., 15: 173-176.

PARÁTIPO, F, Xingu, MT, BR, 11/61, Alvarenga \& Werner, ex Alvarenga.

\section{BRUCHIDAE}

Acanthoscelides ambopygus Kingsolver, 1982, Experientiae, 28: 110-111. HOLÓTIPO, Viçosa, MG, BR, 22/04/81, G.P. Santos.

Acanthoscelides aurulentus Kingsolver, 1985, Entomography, 3: 53-56.

PARÁTIPO, 1, Praia Grande (R.Negro)( $3^{\circ} 00^{\prime} \mathrm{S}, 60^{\circ} 37^{\prime} \mathrm{W}$ ), AM, BR, 10/06/79, M.J.G. Hopkins; 1, idem, 27/06/79, idem; 2, Cachimbo, PA, BR, 08/79, M.J.G. \& H.C. Hopkins.

Acanthoscelides bilobatus Kingsolver, 1982, Experientiae, 28: 112-113.

HOLÓTIPO, Viçosa, MG, BR, 22/04/81, G.P. Santos.

Acanthoscelides bisagittus Kingsolver, 1985, Entomography, 3: 60-61.

PARÁTIPO, 1, Praia Grande (R.Negro) $\left(3^{\circ} 00^{\prime} \mathrm{S}, 60^{\circ} 37^{\prime} \mathrm{W}\right)$, AM, BR, 10/04/79, M.J.G. Hopkins; 1, Reserva Campinas (BR 174, km 44), AM, BR, 13/06/79, M.J.G. Hopkins.

Acanthoscelides cajanae Kingsolver, 1987, Experientiae, 30: 69-71.

HOLÓTIPO, Campinas, SP, BR, s/data, N. Braga.

Acanthoscelides helenae Kingsolver, 1985, Entomography, 3: 56-59.

PARÁTIPO, Reserva Campinas (BR-174, km 44), AM, BR, 19/06/79, M.J.G.Hopkins.

Acanthoscelides hopkinsi Kingsolver, 1985, Entomography, 3: 64-68.

PARÁTIPO, Reserva Campinas (BR-174, km 44), AM, BR, 04/10/79, M.J.G. Hopkins; 2, idem, 13/06/79, idem; 1, idem, 15/06/79, idem; 1, idem, 20/06/79, idem.

Acanthoscelides imitator Kingsolver, 1985, Entomography, 3: 68-69.

PARÁTIPO, 2, BR-316 km 132, PI, BR, 19/09/79, M.J.G. Hopkins; 2, idem, 21/09/79, idem.

Acanthoscelides phalaropus Kingsolver, 1985, Entomography, 3: 61-64.

PARÁTIPO, 2, Praia Grande (R.Negro) $\left(3^{\circ} 00^{\circ} \mathrm{S}, 60^{\circ} 37^{\prime} \mathrm{W}\right)$, AM, BR, 30/05/79, M.J.G. Hopkins; 1, Reserva Campinas (BR-174, km.44), AM, BR, 12/10/79, M.J.G. Hopkins; 1, idem, 22/10/79, idem.

Acanthoscelides unguiculatus Kingsolver, 1982, Experientiae, 28: 107-109.

HOLÓTIPO, Viçosa, MG, BR, 22/04/81, G.P. Santos.

Caryedes cavatus Kingsolver \& Whitehead, 1974, Trans. Am. ent. Soc., 100: 385-387.

PARÁTIPO, 2, Punta Arenas (13 mi E), PU, CR, 10/03/71, D.H. Janzen. Gibbobruchus guanacaste Kingsolver \& Whitehead, 1975, Trans. Am. ent. Soc., 101: 186-190.

PARÁTIPO, 2, Santa Rosa, GU, CR, 19/06/72, D.H. Janzen. 
Mimosetes chrysocosmus Kingsolver, 1985, Entomography, 3: 46-49.

PARÁTIPO, 3, Reserva Campinas (BR-174, Km 44), AM, BR, 06/10/79, M.J.G. Hopkins; 1, idem, 07/10/79, idem; 1, idem, 11/10/79, idem; 1, idem, 14/10/79/ idem; 1 , idem, 24/10/79, idem.

Sennius cupreatus Kingsolver, 1987, Experientiae, 30: 67-69.

HOLÓTIPO, Florestal, MG, BR, 10/11/83, G.P. Santos.

Sennius spodiogaster Kingsolver, 1987, Experientiae, 30: 65-67.

HOLÓTIPO, Florestal, MG, BR, 10/11/84, G.P. Santos.

\section{BUPRESTIDAE}

Agrilus oceanicus Cobos, 1959, Revta bras. Biol., 19: 465.

HOLÓTIPO, M, Ilha de Fernando de Noronha, PE, BR, 05/54, M. Alvarenga, ex Alvarenga; ALÓTIPO, F, idem, idem; PARÁTIPO, 11, idem, idem.

Micrasta alvarengai Cobos, 1959, Revta bras. Biol., $19: 464$.

HOLÓTIPO, F, Ilha de Fernando de Noronha, PE, BR, 05/54, M. Alvarenga, ex Alvarenga.

\section{CARABIDAE}

Helluobrochus ares Reichardt, 1974, Studia ent., 17: 211-302.

PARÁTIPO, F, Araxá, MG, BR, 27/10/65, C. Elias; M, Arapoti, PR, BR, 12/51, A. Maller, ex Alvarenga; M, Jaguariaiva, PR, BR, 30/12/71, F. Giacomel; M, Ponta Grossa, PR, BR, 11/56, F. Justus Jr.; F, Joaçaba, SC, BR, 02/01/67, R. Antoni; F, Nova Teutônia, SC, BR, 02/63, F. Plaumann, ex Maller; M, idem, 10/61, idem.

Helluobrochus bucki Reichardt, 1974, Studia ent., 17: 211-302.

PARÁTIPO, F, Guarapuava, PR, BR, 02/50, H. Schneider.

Helluobrochus capixaba Reichardt, 1974, Studia ent., 17: 211-302.

HOLÓTIPO, M, Santa Teresa, ES, BR, 15/10/66, C.T. \& C. Elias.

Helluobrochus darlingtoni Reichardt, 1974, Studia ent., 17: 211-302.

PARÁTIPO, F, Rondonópolis, MT, BR, 01/63, A. Maller, ex Maller.

Helluobrochus petrus Reichardt, 1974, Studia ent., 17: 211-302.

PARÁTIPO, 2 F, Rio Verde, MT, BR, 10/60, A. Maller, ex Maller.

Helluomorpha araujoi Reichardt, 1974, Studia ent., 17: 211-302.

PARATIPO, F, Rio Verde, MT, BR, 01/67, A. Maller; F, idem, 11/59, idem, ex Maller.

Helluomorpha eulinae Reichardt, 1974, Studia ent., 17: 211-302.

PARATIPO, F, Rio Verde, MT, BR, 11/59, A. Maller, ex Maller.

Pleuracanthus bacchus Reichardt, 1972, Revta bras. Ent., 16: 49-52.

PARÁTIPO, F, Pitanga (S. Jose), PR, BR, 14/08/66, Moure \& Marinoni; 2M, Barueri, SP, BR, 05/05/57, K. Lenko, ex Alvarenga. 


\section{CERAMBYCIDAE}

Acanthoderes comptus Marinoni \& Martins, 1978, Papéis Avulsos Zool., 31: 185-186.

HOLÓTIPO, M, Forte Príncipe da Beira, RO, BR, 11/65, F.M. Oliveira. Acanthoderes lanei Marinoni \& Martins, 1978, Papéis Avulsos Zool., 31: 187-188. HOLÓTIPO, M, Coxim, MT, BR, 11/65, A.Maller; PARÁTIPO, 3, Coxim, MT, BR, 11/65, A. Maller.

Achryson foersteri Bosq, 1953, Comun. zool. Mus. Hist. nat., Montevideo, 4: 1-4. PARÁTIPO, M, Entre Rios, AR, 12/40.

Aleiphaquilon plaumanni Martins, 1975, Papéis Avulsos Zool., 29: 7-20.

PARÁTIPO, F, Guaraúna, PR, BR, 10/37, F. Justus Jr..

Alphus capixaba Marinoni \& Martins, 1978, Papéis Avulsos Zool., 31: 178-179.

PARÁTIPO, 2, Linhares, ES, BR, 10/72, P.C. Elias.

Ametysphaerion nigripes Martins \& Monné, 1974, Papéis Avulsos Zool., 28: 269-293.

PARÁTIPO, F, Foz do Iguaçu, PR, BR, 07/12/66, Exc. Dep. Zool. UFPR. Aneuthetochorus conjunctus Napp \& Martins, 1984, Revta bras. Ent., 28: 51-58. PARÁTIPO, M, Linhares, ES, BR, 22-31/10/73, C.T. \& C. Elias.

Cacostola acuticauda Marinoni \& Martins, 1982, Revta bras. Ent., 26: 248-249.

PARÁTIPO, F, Córrego do Itá, ES, BR, 11/56, W. Zikán; F, Linhares, ES, BR, 11/72, P.C. Elias.

Cacostola cana Marinoni \& Martins, 1982, Revta bras. Ent., 26: 249-250.

HOLÓTIPO, M, Linhares, ES, BR, 21-30/10/73, C.T. \& C. Elias.

Callia flavipes Zajciw, 1958, Revta bras. Ent., 8: 55-57.

PARATIPO, F, Rio de Janeiro (Corcovado), RJ, BR, 03/11/53, D. Zajciw, ex Zajciw.

Callisema socium Martins \& Galileo, 1990, Revta bras. Ent., 34: 477-478.

PARÁTIPO, 8 F, Linhares (Pq.Sooretama), ES, BR, 11/67, F.M. Oliveira;

F, Guaraúna, PR, BR, 1940, F. Justus Jr.; 2 F, Nova Teutônia, SC, BR, 10/66,

F. Plaumann; M, idem, idem; 2 F, idem, 10/72, idem; 4 M, Linhares (Pq. Sooretama), ES, BR, 11/67, F.M. Oliveira; M, Ponta Grossa, PR, BR, 03/44, M. Liming.

Cathexis vitticollis Zajciw, 1967, An. Acad. bras. Ci., 39: 322-324.

PARÁTIPO, F, Linhares (Pq. Sooretama), ES, BR, 12/11/60, D. Zajciw, ex

Zajciw; M, idem, 14/11/60, idem.

Cephaloplon pedunculatum Martins \& Napp, 1986, Revta bras. Ent., 30: 227-241.

PARÁTIPO, M, Morne Bleu (2700'), TR, TB, 23/08/69, H. \& A. Howden.

Chaetosopus contiguus Napp \& Martins, 1988, Revta bras. Ent., 32: 479-486.

HOLÓTIPO, F, Curitiba (Cascatinha), PR, BR, 15/12/68, S. Laroca.

Chaetosopus infalsatus Napp \& Martins, 1988, Revta bras.Ent., 32: 479-486.

PARÁTIPO, F, Passa Quatro (Faz.Campos), MG, BR, 30/12/17, J.F. Zikán.

Clausirion comptum Martins \& Napp, 1982, Dusenia, 13: 59-64. 
PARÁTIPO, M, Sinop (BR-163, Km 500-600), MT, BR, 10/74, Alvarenga \& Roppa.

Colobothea rubroornata Zajciw, 1962, An. Acad. bras. Ci., 34: 285-287.

PARÁTIPO, M, Linhares (Pq. Sooretama), ES, BR, 17-21/11/60, D. Zajciw, ex Zajciw.

Colobothea seriatomaculata Zajciw, 1962, An. Acad. bras. Ci., 34: 283-284.

PARÁTIPO, F, Rio de Janeiro (Corcovado), RJ, BR, 01/12/57, D. Zajciw, ex Zajciw.

Compsibidion concisum Napp \& Martins, 1985, Papéis Avulsos Zool., 36: 131-146.

PARÁTIPO, F, Linhares, ES, BR, 9-15/01/73, C. Elias; M, Condeúba, BA, BR, 02/76, S. Souza.

Compsibidion derivativum Martins, 1971, Arq. Zool., S. Paulo, 16: 1343-1508.

HOLÓTIPO, M, Cáceres, MT, BR, 19/10/61, F.M. Oliveira.

Compsibidion triviale Napp \& Martins, 1985, Papéis Avulsos Zool., 36: 131-146.

PARÁTIPO, F, Poços de Caldas (Morro do Ferro), MG, BR, 01/11/70, J.

Becker.

Compsocerus deceptor Napp, 1976, Revta bras. Ent., 20: 1-64.

PARÁTIPO, F, Virgínia (Faz. Campos, 1500m), MG, BR, 07/12/19, J. Zikán; M, idem, 25/11/15, idem; M, S. José do Barreiro (Serra da Bocaina), SP, BR, 12/70, P.S. Motta.

Compsocerus proximus Napp, 1977, Dusenia, 10: 97-100.

HOLÓTIPO, M, Ponta Grossa (Pedreira), PR, BR, 12/42, F. Justus Jr..

Cycnidolon pumillum Napp \& Martins, 1985, Papéis Avulsos Zool., 36: 131-146.

PARÁTIPO, 2 F, Pedra Azul (700 m), MG, BR, 11/72, Seabra \& Oliveira.

Dilocerus marinonii Napp, 1980, Revta bras. Ent., 24: 193-196.

PARÁTIPO, M, Cochabamba, CO, BO.

Eburia abacta Martins, 1981, Papéis Avulsos Zool., 34: 205-219.

HOLÓTIPO, M, Conceição da Barra, ES, BR, 26/08/69, C.T. \& C. Elias;

PARÁTIPO, F, idem, idem; F, Santa Teresa, ES, BR, 12/12/66, C.T. \& C.

Elias; 2 F, idem, 22/10/66, idem; F, idem, 29/10/66, idem; M,idem, 04/12/66.

Eburodacrys alini Napp \& Martins, 1980, Papéis Avulsos Zool., 33: 77-97.

PARÁTIPO, F, Santa Teresa, ES, BR, 08/01/66, C.T. \& C. Elias; F, S. Bernardo do Campo, SP, BR, 22/03/63, R. Grantsau; M, idem, 22/03/63, idem.

Eburodacrys lenkoi Napp \& Martins, 1980, Papéis Avulsos Zool., 33: 77-97.

PARÁTIPO, $1 \mathrm{~F}, 1 \mathrm{M}$, Viçosa, MG, BR, s/data, E. Amante; F, Barueri, SP, BR, 18/11/54, K. Lenko; M, idem, SP, BR, 11/66, K. Lenko.

Eburodacrys obscura Martins, 1973, Papéis Avulsos Zool., 26: 201-213.

PARÁTIPO, M, Botucatu, SP, BR, 08/70, O.J. Chamma.

Eburodacrys seabrai Zajciw, 1958, Revta bras. Ent., 8: 233-262.

PARÁTIPO, F, Soledade (Juazeirinho), PB, BR, 29/03/56, A.G.A. Silva, ex 


\section{Zajciw.}

Ectenessa melanicornis Napp \& Martins, 1981, Papéis Avulsos Zool., 34:349-401.

HOLÓTIPO, M, Linhares, ES, BR, 11/65, A. Maller; PARÁTIPO, 2 F, Santa Teresa, ES, BR, 06/11/66, C.T. \& C.Elias; 2 F, idem, 12/12/66, idem; M, Linhares, ES, BR, 12/65, A. Maller; M, Santa Teresa, ES, BR, 04/12/66, C.T. \& C. Elias; 2 M, idem, 21/11/66, idem.

Elaphopsis earinus Martins \& Napp, 1989, Revta bras. Ent., 33: 57-65.

HOLÓTIPO, M, Conceição da Barra, ES, BR, 25/09/69, C.T. \& C. Elias.

Epropetes atlantica Martins, 1975, Papéis Avulsos Zool., 29: 7-20.

PARÁTIPO, M, Guaraúna, PR, BR, 10/37, F. Justus Jr.

Epropetes hirsuta Martins \& Napp, 1984, Revta bras. Ent., 28: 435-436.

HOLÓTIPO, M, Represa Rio Grande, RJ, BR, 02/61, F.M. Oliveira;

PARÁTIPO, F, Rio de Janeiro (Corcovado), RJ, BR, 03/12/65, Moure \& Seabra.

Epropetes serrana Martins \& Napp, 1984, Revta bras. Ent., 28: 434-435.

PARÁTIPO, M, Mar de Hespanha, MG, BR, 14/11/09, J.F. Zikán.

Epropetes zonula Martins \& Napp, 1984, Revta bras. Ertt., 28: 437-438.

PARÁTIPO, M, Virgínia (Faz.Campos, 1500 m), MG, BR, 01/12/20, J.F.Zikán.

Ethemon basirufum Napp, 1979, Revta bras. Biol., 39: 901-917.

PARÁTIPO, F, Nova Friburgo, RJ, BR, 1947, H. Zellibor; M, Friburgo, RJ, BR, 02/1934, Oiticica.

Ethemon lepidum rufofemorale Napp, 1979, Revta bras. Biol., 39: 901-917.

HOLÓTIPO, M, Curitiba, PR, BR, 09/02/72, D.S. Napp; PARÁTIPO, F, Curitiba, 03/03/68, Dep.Zool.; F, 09/02/72, D.S. Napp; F, Lapa, PR, BR, 03/73, F. Giacomel; 5 M, 3 F, 17/03/70, Marinoni \& Moure; F, Ponta Grossa, PR, BR, 05/44, F. Justus Jr.; M, 04/43, idem; F, Fragosos, SC, BR, 03/03/68, R.C. Marinoni; F, idem, 09/04/68, idem; 1 F, 1 M, Mafra, SC, BR, 12/68, A. Maller; F, S. Bento doSul (Rio Vermelho), SC, BR, 05/74, Rank; M, S. Bento do Sul, SC, BR, 15/03/74, A. Maller; M, S. Bento do Sul (Rio Vermelho), SC, BR, 04/74 Rank.

Falsamblesthis ibiyara Marinoni, 1978, Revta bras. Ent., 22: 91-94.

PARÁTIPO, F, Itu (Faz. Pau d'Alho), SP, BR, 9-10/11/61, L.R. Silva; M,

Passa Quatro (Faz. Campos), MG, BR, 03/02/21, J.F. Zikán.

Gnomidolon meridanum Napp \& Martins, 1985, Papéis Avulsos Zool., 36: 111-130.

PARÁTIPO, M, Lagunillas (1000m), ME, VE, 19-31/05/77, Bordon.

Gnomidolon opacicolle Napp \& Martins, 1985, Papéis Avulsos Zool., 36: 111-130.

PARÁTIPO, F, Sinop (BR-163, Km 500-600), MT, BR, 10/76, Roppa \& Alvarenga.

Gorybia suturella Martins, 1976, Arq. Zool., S. Paulo, 27: 165-370. 
PARÁTIPO, F, Coxim, MT, BR, 10/69, A. Maller.

Gounellea franzae Lane, 1970, Studia ent., 13: 378-380.

PARATIPO, F, Forte Príncipe da Beira, RO, BR, 07/11/61, F.M. Oliveira;

$\mathrm{M}$, idem, 07/11/61, idem.

Haenkea zischkai Tippmann, 1953, Dusenia, 4: 222.

PARÁTIPO, $1 \mathrm{~F}, 1 \mathrm{M}$, Chapare (400m), CO, BO, 08/50, R. Zischka, ex Justus.

Hesperophymatus limexylon Zajciw, 1959, An. Acad. bras. Ci., 31: 605-616.

PARÁTIPO, F, Teresópolis, RJ, BR, 18/11/55, D. Zajciw, ex Zajciw; M, idem, 09/11/58, idem.

Hesycha bimaculata Martins \& Galileo, 1990, Papéis Avulsos Zool., 37: 76.

PARÁTIPO, M, Santa Teresa, ES, BR, 06/11/66, C.T. \& C. Elias.

Heterachthes fraterculus Martins \& Napp, 1986, Revta bras. Ent., 30: 227-241.

PARÁTIPO, F, Puntarenas (50m), PU, CR, 05/12/72, V.O. Becker; F, Comitan (31 mi. SE), CH, ME, 18-19/06/65, Burke, Meyer, Shaffner; M, Canas (4 km N), GU, CR, 30/06/66, D. Veirs; M, La Pacifica (4 km NW Canas), GU, CR, 10/06/71, F. Cordero.

Heterachthes wappesi Martins \& Napp, 1986, Revta bras. Ent., 30: 227-241.

PARÁTIPO, F, Corrillo Puerto, QR, ME, 27/09/73, V.O. Becker.

Hexoplon fernuginosum Martins, 1959, Papéis Avulsos Dep. Zool., 13: 347-349.

PARÁTIPO, 6, Marilia, SP, BR, 11/45, Zellibor.

Hippopsis pertusa Galileo \& Martins, 1988. Revta bras. Ent., 32: 182-184.

PARATIPO, M, Brusque, SC, BR, 28/12/69, V.O. Becker; M, Corupá, SC, BR, 11/67, A. Maller; M, S. Bento do Sul (Rio Vermelho) SC, BR, 05/74, Rank.

Hippopsis solangeae Carvalho, 1981, Revta bras. Ent., 25: 325-331.

PARÁTIPO, M, Represa Rio Grande, RJ, BR, 03/02/67, F.M. Oliveira; M, Barueri, SP, BR, 11/65, K. Lenko.

Hoplistocerus lanei Zajciw, 1960, Revta bras. Ent., 9: 146-149.

PARÁTIPO, M, Rio de Janeiro (Corcovado), RJ, BR, 30/09/55, D. Zajciw, ex Zajciw.

Hypsioma renatoi Martins \& Galileo, 1990, Papéis Avulsos Zool., 37: 56.

PARÁTIPO, F, Linhares, ES, BR, 01/69, A. Maller; F, idem, 12/65, idem; F, Linhares (Pq. Sooretama), ES, BR, 19/11/68, C. Elias; M, idem, 17/11/67, F.M. Oliveira.

Jampruca nigricornis Napp \& Martins, 1982, Papéis Avulsos Zool., 34:349-401.

PARÁTIPO, F, Baixo Guandu, ES, BR, 09/12/70, C. Elias.

Jampruca tyligma Napp \& Martins, 1982, Papéis Avulsos Zool., 34: 349-401.

PARÁTIPO, F, Encruzilhada (Rod.Rio-Bahia,Km.965), BA, BR, 11/70, Roppa.

Mephritus genuinus Napp \& Martins, 1982, Revta bras. Ent., 26: 75-86.

PARATIPO, F, Morretes (Marumbi, 500m), PR, BR, 14/02/67, Laroca \& 
Giacomel; M, idem, 15-16/02/69, idem.

Mephritus guttatus Napp \& Martins, 1982, Revta bras. Ent., 26: 75-86.

PARÁTIPO, M, Pedra Azul, MG, BR, 11/70, Seabra \& Oliveira.

Montesia fasciolata Martins \& Galileo, 1990, Revta bras. Ent., 34: 586.

HOLÓTIPO, M, Turrialba, CA, CR, 1-15/08/62, V.O. Becker.

Myoxomorpha seabrai Marinoni \& Dalossi, 1971, Arq. Mus. Nacional, R. Janeiro, 54: 190-191.

PARÁTIPO, F, 2 M, Cachimbo, PA, BR, 25/9-10/56, Travassos-Oliveira \& Adão.

Myzomorphus flavipes Galileo, 1987, Revta bras. Ent., 31: 595-597.

HOLÓTIPO, F, Conceição da Barra, ES, BR, 29/11/69, C.T. \& C. Elias.

Neocompsa bimaculata Martins \& Napp, 1986, Revta bras. Ent., 30: 227-241.

PARÁTIPO, F, La Pacifica (4 km NW Canas), GU, CR, 22-26/05/84, Ryley,

Rider \& Le Doux.

Neocompsa limatula Martins \& Napp, 1986, Revta bras. Ent., 30: 227-241.

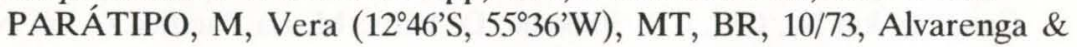
Roppa.

Nesostizocera jassuara Martins \& Napp, 1983, Papéis Avulsos Zool., 35: 79-92.

PARÁTIPO, 1 F, 2 M, Baixo Guandu, ES, BR, 10/71, P.C. Elias.

Nesozineus bisignatus Hoffmann, 1984, Revta bras. Ent., 28: 543-544.

PARÁTIPO, M, Condeúba, BA, BR, 11/12/1888, Gounelle.

Nesozineus confusus Marinoni \& Martins, 1978, Papéis Avulsos Zool., 31:

181-182.

HOLÓTIPO, F, Baixo Guandu, ES, BR, 05/1972, F. Giacomel; PARÁTIPO, $2 \mathrm{~F}$, idem, idem.

Nesozineus griseolus Hoffmann, 1984, Revta bras. Ent., 28: 539-540.

PARÁTIPO, F, S. Luzia (Brandão dos Chandoca), PB, BR, 26/03/56, A.G.A. Silva.

Nesozineus obscurus Hoffmann, 1984, Revta bras. Ent., 28: 541.

PARÁTIPO, F, Villa Angela, CH, AR, 02/37, Mallo.

Niophis bucki Martins \& Monné, 1973, Revta bras. Ent., 17: 19-27.

HOLÓTIPO, M, Santa Teresa, ES, BR, 15/10/66, C.T. \& C. Elias; PARÁTIPO, F, idem, idem.

Nyctonympha cicatricosa Zajciw, 1968, Revta bras. Biol., 28: 423-424.

PARÁTIPO, 1, Rio de Janeiro (Corcovado), RJ, BR, 03/11/53, D. Zajciw, ex Zajciw.

Ochrus duplicatus Napp \& Martins, 1982, Papéis Avulsos Zool., 34: 349-401.

PARÁTIPO, M, Sinop (BR-163, Km 500-600), MT, BR, 10/74, Alvarenga \& Roppa.

Ommata (Chariergoides) carinicollis Zajciw, 1963, An. Acad.b ras. Ci., 35: 241-255.

PARÁTIPO, F, Rio de Janeiro (Corcovado), RJ, BR, 09/10/58, Zajciw, ex 
Zajciw; F, idem, 26/09/58, idem.

Ommata (Chariergoides) flava Zajciw, 1963, An. Acad. bras. Ci., 35: 241-255.

PARÁTIPO, M, Rio de Janeiro (Corcovado), RJ, BR, 04/10/54, D. Zajciw, ex Zajciw.

Oncideres boliviana Heyrowsky, 1952, Acta Soc. Ent. Cechosl., 49: 44-45, ex Justus.

PARÁTIPO, 1 M, 1F, Cochabamba, CO, BO, 03/52, Heyrowsky.

Oncideres captiosa Martins, 1981, Papéis Avulsos Zool., 34: 229-230.

PARÁTIPO, 1, Porto Bemberg, MI, AR, 11/42, F. Justus Jr.; 1, Condeúba, BA, BR, 02/76, S. Souza; 1, Linhares, ES, BR,01/67, A. Maller; 2, idem, 06/01/71, C. Elias; 1, idem, 10/65, A. Maller; 7, idem, 11-12/65, idem; 1, idem, 12/67, idem; 1, Linhares (Pq. Sooretama), ES, BR, 07/11/64, Oliveira et alii; 12, idem, 7/11/67, idem; 3, Foz do Iguaçu, PR, BR, 07/12/66, D. Zool. UFPR.; 1, Tibagi (Salto Conceição), PR, BR, 11/56, F. Justus Jr.; 1, idem, 12/57, idem; 1, Santa Maria, RS, BR, 26/12/70, I.Coronel; 3, Joinville, SC, BR, 10/66, H. Miers; 2, idem, 11/65, idem; 1, idem, 12/65, A. Maller; 1, idem, 12/66, idem; 1, Nova Teutônia, SC, BR, 07/73, F. Plaumann; 1, idem, 12/66, idem; 3 , idem, $12 / 72$, idem.

Oreodera candida Marinoni \& Martins, 1978, Papéis Avulsos Zool., 31: 177-178. PARÁTIPO, 1, Represa Rio Grande, RJ, BR, 03/61, F.M.Oliveira; 1, idem, 10/64, idem; 1, Rio de Janeiro (Corcovado), RJ, BR, 03/12/65, Moure \& Seabra.

Oreodera macropoda Marinoni \& Martins, 1978, Papéis Avulsos Zool., 31: 175-176.

HOLÓTIPO, M, Rio Branco, AC, BR, 15-20/11/65, F.M. Oliveira; PARÁTIPO, $3 \mathrm{M}$, idem, idem.

Oreodera tijuca Marinoni \& Martins, 1978, Papéis Avulsos Zool., 31: 174-175.

PARÁTIPO, M, Rio de Janeiro (Corcovado), RJ, BR, 13/02/58, C.A.C. Seabra.

Orthoschema (Orthoschema) erythrocephala Napp \& Martins, 1988, Revta bras. Ent., 32: 479-486.

HOLÓTIPO, M, Rolândia, PR, BR, 10/53, Diringshofen; PARÁTIPO, F, Curitiba, PR, BR, 10/10/71, S. Laroca; 1 M, idem, 11/11/67, D. Zoo. UFPR, idem; F, idem, 12/46, F. Justus Jr.; F, Guarapuava, PR, BR, 20/01/72, Mielke \& Tangerini; F, Ponta Grossa, PR, BR, 1940, F. Justus Jr.; M, idem, 12/43, idem; F, Rolândia, PR, BR, 11/51, A. Maller; 1 F, 1 M, Nova Teutônia, SC, BR,11/55, F. Plaumann; 2 F, 2 M, idem, 12/65, idem; F, idem, 21/11/65, idem; M, idem, 11/66, idem; $M$, idem, 02/74, idem.

Paromoeocerus stictonotus Napp, 1976, Revta bras. Ent., 20: 1-64.

PARÁTIPO, F, Itatiaia (Maromba), RJ, BR, 15/01/25, J.F. Zikán; M, idem, 29/12/52, Leite, Seabra \& Zikán.

Periboeum guttigerum Napp \& Martins, 1984, Papéis Avulsos Zool., 35: 125-133. HOLOTIPO, F, Santa Teresa, ES, BR, 21/11/66, C.T. \& C. Elias. 
Piezarina smaragdina Martins, 1976, Arq. Zool., S. Paulo, 27: 165-370.

PARÁTIPO, F, Coxim, MT, BR, 10/69, A. Maller.

Piola quiabentiae Marinoni, 1974, Revta bras. Ent., 18: 86-91.

HOLÓTIPO, F, Morillo, SA, AR, 12/72, H. Zimmermann; PARÁTIPO, 2

F, idem, idem; $M$, idem 28/11/73, idem.

Pseudogisostola reichardti Fontes \& Martins, 1977, Revta bras. Ent., 21: 13-14.

PARÁTIPO, F, Linhares, ES, BR, 04/12/71, A.C. Domingos; M, Linhares (Pq. Sooretama), ES, BR, 11/67, F.M. Oliveira.

Pucallpa robusta Monné, 1978, Revta bras. Biol., 38: 587-593.

PARÁTIPO, F, Sinop (BR-163, Km 500-600), MT, BR, 10/75, Roppa \& Alvarenga; M, idem, 10/74, Alvarenga \& Roppa.

Pygmodeon puniceum Martins, 1970, Arq. Zool., S. Paulo, 16: 1151-1342.

PARÁTIPO, M, Balzapampa (Ruta de Quito), BO, EQ, 09-10/1893, M. de Mathan.

Rhaphiptera triangulifera Lane, 1974, Revta bras. Ent., 18: 93-99.

PARÁTIPO, 2F, Linhares, ES, BR, 11/72, B. Silva.

Rhopalophora paraensis Martins \& Napp, 1989, Revta bras. Ent., 33: 57-65.

PARÁTIPO, M, Serra Norte (Rio Salobo), PA, BR, 16/05/84; M, idem, 19-22/05/84, idem.

Rhopalophora smaragdina Martins \& Napp, 1989, Revta bras. Ent., 33: 57-65.

HOLÓTIPO, M, Itapina, ES, BR, 26-30/12/67, C.T. Elias; PARÁTIPO, 3

F, 2 M, idem, idem; 3 F, 1 M, idem, 18-23/12/67, idem; F, Dourados, MS,

BR, 01/76, J. Lorenzoni.

Smodicum longicorne Martins, 1975, Arq. Zool., S. Paulo, 26: 319-359.

PARÁTIPO, F, Santa Teresa, ES, BR, 22/10/66, C.T. \& C. Elias.

Sphaerioeme rubristerna Martins \& Napp, 1992, Revta bras. Zool., 7(4): 474.

PARÁTIPO, F, M, Coxim, MT, BR, 10/69, A. Maller; F, Rio Brilhante, MT, BR, 21-28/10/70, V.O. Becker; F, Sete Lagoas (IPEACO), MG, BR, 14/02/59, C. Biezanko; M, Piracicaba, SP, BR, 31/10/69, D. Link; 38 F, 39 M, Porecatu, PR, BR, 20/10/70, Becker \& Hatschbach.

Spinoplon bicolor Napp \& Martins, 1985, Papéis Avulsos Zool., 36: 111-130.

PARÁTIPO, F, Encruzilhada (Rod. Rio-Bahia, Km 965), BA, BR, 11/74,

Seabra \& Roppa.

Spinoplon inusitatum Napp \& Martins, 1985, Papéis Avulsos Zool., 36: 111-130.

PARÁTIPO, F, Encruzilhada (Rod. Rio-Bahia,Km 965), BA, BR, 11/72, Seabra \& Roppa.

Stenoeme kempfi Martins, 1980, Papéis Avulsos Zool., 33: 221-229.

PARÁTIPO, F, Linhares, ES, BR, 27/11/72, C. Elias; M, idem, 15-20/10/73, idem.

Stizocera juati Martins \& Napp, 1983, Papéis Avulsos Zool., 35: 79-92.

PARÁTIPO, F, Morro da Garça, MG, BR, 18-20/10/64, Exp. Dep. Zool.

Stizocera mojuba Martins \& Napp, 1983, Papéis Avulsos Zool., 35: 79-92. 
PARÁTIPO, 3 F, 1 M, Linhares, ES, BR, 23-31/10/73, C. Elias; M, idem, 16-22/01/73, idem; 2 M, Santa Teresa, ES, BR, 12/12/66, C.T. \& C. Elias; M, idem, 28/11/66, idem.

Stizocera seminigra Martins \& Napp, 1983, Papéis Avulsos Zool., 35: 79-92.

PARÁTIPO, 2 F, 2 M, Cachimbo, BA, BR, 1890, Pujol.

Tapeina coronota integra Marinoni, 1972, Bol. Univ. Fed. Paraná, 5: 20-23.

HOLÓTIPO, M, Ortigueira (Monjolinho), PR, BR, 01/43, F. Justus Jr.; ALÓTIPO, F, idem, idem; PARÁTIPO, M, idem, 01/44, idem; 5 F, Passos, MG, BR, 9-15/10/63, C. Elias; 1 F, 1 M, Guaraúna PR, BR, 1940, F. Justus $\mathrm{Jr}$. .

Tapeina erectifrons amazona Marinoni, 1972 Bol. Univ. Fed. Paraná, 5: 55-57.

PARÁTIPO, 2 F, Rio Branco, AC, BR, 15-20/11/61, F.M. Oliveira; 1 F, 1 M, Manaus, AM, BR, 08/59, C. Elias.

Tapeina rubronigra Marinoni, 1972, Bol. Univ. Fed. Paraná, 5: 39-43.

ALÓTIPO, F, Rio Branco, AC, BR, 15-20/11/61, F.M. Oliveira; PARÁTIPO, 5 F, $1 \mathrm{M}$, idem, idem.

Tapeina rudifrons Marinoni, 1972, Bol. Univ. Fed. Paraná, 5: 30-34.

PARÁTIPO, M, Linhares, ES, BR, 11/66, A. Maller.

Tapeina transversifrons brevifrons Chemsak \& Linsley, 1972, in Bol. Univ. Fed.

Paraná, 5: 64-67.

PARÁTIPO, M, Venedio (=Venadillo), SI, ME, 30/06/18, Van Dyke Ex CIS.

Tapeina transversifrons centralis Marinoni, 1972, Bol. Univ. Fed. Paraná, 5:62-67.

PARÁTIPO, 1 F, 1 M, Tamanique (Tamanique), LI, ES, 04/05/60, J. Bechyné, ex Seabra;1 F, 1 M, La Chorrera, PA, PA, 23/05/44, K.E. Frick, ex CIS.

Tapuruia jolyi Napp \& Martins, 1985, Papéis Avulsos Zool., 36: 111-130.

PARÁTIPO, F, Granja Exp., ZU, VE, 21/05/61, J.R. Labrador.

Taurorcus chabrillacii Thomson, 1857, in Revta Floresta,1: 53-55.

ALÓTIPO, F, Pinhão, PR, BR, 11/66, Moure,Mielke \& Marinoni.

Taurorcus mourei Marinoni, 1969, Revta Floresta, 1: 60-61.

HOLÓTIPO, F, Guarapuava, PR, BR, 01/57, H. Schneider.

Temnopis latifascia Martins \& Monné, 1975, Papéis Avulsos Zool., 28: 269-293. PARÁTIPO, 2 F, Ibiá, MG, BR, 20/10/63, C.T. \& C.Elias; F, Jaguariaiva, PR, BR, 24/12/69, F. Giacomel; M, Ibiá, MG, BR, 20/10/63, C.T. \& C. Elias; 3M, Passos, MG, BR, 10/1963, C.T. \& C. Elias; M, Curitiba, PR, BR, 1965, S. Takashima; M, Foz do Iguaçu, PR, BR, 07/12/66, Exc. D. Zool. UFPR.

Tropidion cruentum Martins \& Napp, 1986, Revta bras.Ent., 30: 5-12.

PARÁTIPO, M, Sinop (BR-163, Km 500-600), MT, BR, 10/75, Roppa \& Alvarenga; M, Vera (12 $\left.46^{\circ} \mathrm{S}, 55^{\circ} 36^{\prime} \mathrm{W}\right)$, MT, BR, 10/73, Alvarenga \& Roppa.

Tropidion pubicolle Martins \& Napp, 1986, Revta bras. Ent., 30: 5-12.

PARÁTIPO, F, Jutai (BR 319, Km 369), AM, BR, 09/78, Silva \& Becker; 
M, Tucurui, PA, BR, 01/80, B.Silva.

Xenocrasis vestitipennis Zajciw, 1963, An. Acad. bras.Ci., 35: 241-255.

PARÁTIPO, F, Rio de Janeiro (Corcovado), RJ, BR, 25/10/53, D. Zajciw, ex Zajciw; M, idem, 29/11/58, idem.

\section{CHRYSOMELIDAE}

Alagoasa condensa Bechyné, 1956, Ent. Arb., 7: 1052.

PARÁTIPO, 1, Campos do Jordão, SP, BR, 11/06, Luederwaldt, ex Alvarenga; 1, S. Paulo (Ipiranga), SP, BR, s/data, ex Alvarenga.

Alagoasa parana Samuelson, 1985, Revta bras. Ent., 29: 579-585.

HOLÓTIPO, M, Ponta Grossa (Vila Velha), PR, BR, 08/12/79, J.A. Winder; PARÁTIPO, 2, idem, 02/75, idem; 1, idem, 09/04/81, idem; 1, idem, 10/04/81, idem; 4, idem, 17/11/80, idem.

Anacassis phaeopoda Buzzi, 1976, Dusenia, 9: 107-111.

HOLÓTIPO, 1, S. José dos Pinhais (BR-277, Km 25), PR, BR, 21/04/76, Z. Buzzi; PARÁTIPO, 17, idem, idem: 1, Curitiba, PR, BR, 20/02/74, P. McFadyen; 2, Corupá, SC, BR, 12/41, A. Maller.

Aristonoda pereirai Bechyné, 1958, Ent. Arb., 9: 485.

PARÁTIPO, 4, Pouso Alegre, MG, BR, 12/53, F.S. Pereira, ex Alvarenga. Brasilaphtona eredia Bechyné, 1956, Ent. Arb., 7: 972.

PARÁTIPO, 1, Campos do Jordão, SP, BR, 1906, Luederwaldt, ex Alvarenga.

Cephaloleia bucki Uhmanm, 1957, Beitr. Ent., 7: 36.

PARÁTIPO, M, Vila Oliva, RS, BR, 08/02/52, P. Buck, ex Plaumann.

Cephaloleia linke; Uhmann, 1939, Arb. morph. taxon. Ent. Berl., 6: 153.

PARÁTIPO, M, Nova Teutônia, SC, BR, 18/10/35, F. Plaumann, ex Plaumann.

Chlamisus brittocostai Bokermann, 1962, Studia ent., 5: 277-288.

PARÁTIPO, F, S. Bernardo do Campo, SP, BR, 19/10/60, W. Bokermann, ex Alvarenga.

Chlamisus chapadensis Bokermann, 1962, Revta bras. Biol., 22: 153-156.

PARÁTIPO, F, Chapada dos Guimarães, MT, BR, 24-29/10/61, F.M.

Oliveira, ex Alvarenga.

Chrysostetha adippe disjuncta Bechyné, 1950, Revta Ent., Rio de J., 21: 115-156.

CÓTIPO, 1, Guandu, ES, BR, 13/12/20, F. Hoffmann, ex Mus. Nacional.

Colaspis bondari Bechyné \& Bechyné, 1968, Mem. Soc. Cienc. nat. La Salle, 28 (81): 240.

PARÁTIPO, 1, s/local., BA, BR, G.Bondar, ex Mus. Nacional.

Cosmogramma wygodzinskyi Bechyné, 1948, Revta Ent., Rio de J., 19: 295-312.

CÓTIPO, 1, Itatiaia (Faz. Penedo), RJ, BR, 01/11/42, Wygodzinsky, ex Mus. Nacional.

Diabrotica eruptiva Bechyné, 1955, Inst. Sci. Nat. Belg., 31: 11. 
PARÁTIPO, 1, Marcelino Ramos, RS, BR, 15/10/39, ex Alvarenga.

Dorysterna melzeri Bechyné, 1950, Revta Ent., Rio de J., 21: 115-156.

CÓTIPO, 1, S. Paulo (Saúde), SP, BR, 08/12/19.

Eumolpus episternalis Springlova, 1960, Mem. Inst.r. Sci. nat. Belg., (2) 60: 63.

PARÁTIPO, 1, Pedra, PE, BR, s/data, ex Alvarenga; 2,Natal (Parnamirim),

RN, BR, 10/50, M. Alvarenga, ex Alvarenga.

Eumolpus franciscus fortis Springlova, 1960, Mem.Inst.r.Sci. nat. Belg., (2)60: 30.

PARÁTIPO, 1, Vila Bela, MT, BR, 03/53, M. Braz, ex Alvarenga.

Eumolpus incisellus Springlova, 1960, Mem. Inst.r. Sci. nat. Belq., (2) 60: 22.

HOLÓTIPO, Martins, RN, BR, 19/03/50, P. Fonseca, ex Alvarenga;

ALÓTIPO, idem, idem.

Eumolpus itatiaiensis Springlova, 1960, Mem. Inst.r. Sci. nat. Belg., (2) 60: 34 .

PARÁTIPO, 2, Itatiaia, RJ, BR, 04/11/32, W.Zikán, ex Mus. Nacional; 1, idem, 16/11/29, idem.

Eumolpus truncatus Springlova, 1960, Mem.I nst.r. Sci. nat. Belg., (2) 60: 22.

PARÁTIPO, 1, Santos, SP, BR, 1915, ex Mus. Nacional.

Galerucella pereirai Bechyné, 1955, Ent. Arb., 7: 300.

PARÁTIPO, 2, Silveiras, SP, BR, 01/54, F.S. Pereira, ex Alvarenga.

Hibosispa nitida Uhmann, 1939, Arb. morph. taxon. Ent. Berl., 6: 154.

PARÁTIPO, 1, Nova Teutônia, SC, BR, 02/11/39, F. Plaumann, ex Plaumann.

Lioplacis caratubae Buzzi, 1977, Dusenia, 10: 229-232.

HOLÓTIPO, S. José dos Pinhais (BR-277, Km 25), PR, BR, 12/02/76, Z.

Buzzi; PARÁTIPO, 1, idem, 04/07/76, idem; 1, idem, 05/07/76, idem; 2, idem, 12/02/76, idem; 1, idem, 17/01/76, idem; 1, idem, 20/06/76, idem; 1, idem, 23/08/76, idem; 1 , idem, 30/06/76, idem; 1 , idem, 30/11/76, idem; 2,idem, 30/12/76, idem.

Longeumolpus batesi benjaminus Springlova, 1960, Mem. Inst.r. Sci. nat. Belg.,

(2) 60: 17.

PARÁTIPO, 1, Benjamin Constant, AM, BR, 06/42, A.Parko, ex Alvarenga. Luperodes pereirai Bechyné, 1957, Ent.Arb., 7: 326.

PARÁTIPO, 4, El Porton, BO, 02/03/54, C.Gans \& F.Pereira, ex Alvarenga.

Lyraletes minor minimus Bechyné, 1953, Ent. Arb., 4: 110.

PARÁTIPO, 1, Nova Teutônia, SC, BR, 10/34, ex Mus.Nacional.

Maecolaspis cribricollis machacalisa Bechyné, 1958, Ent. Arb., 9: 503.

PARÁTIPO, 1, Machacalis, MG, BR, 12/54, ex Alvarenga.

Neothona undecima Bechyné, 1959, Beitr. neotrop. Fauna, 1: 272.

PARÁTIPO, 1, Cochabamba, CO, BO, 12/53, M. Alvarenga, ex Alvarenga;

35, Campo Grande, MS, BR, 08/11/52, M. Alvarenga, ex Alvarenga.

Piobuckia cyanescens Bechyné, 1956, Ent. Arb., 7:1 006.

PARÁTIPO, 1, Campos do Jordão, SP, BR, 11/06, Luederwaldt, ex Alvarenga. 
Plagiometriona palmeirensis Buzzi, 1992, Revta bras. Zool., 7: 215-216.

HOLÓTIPO, El Limon (1300m), Litoral, DF, VE, 20/06/65, Bordon; PARÁTIPO, 5, idem, 19/06/65, idem; 1,idem, 20/06/65, idem.

Stichotaenia alvarengai Bechyné, 1954, Ent. Arb., 5: 634.

PARÁTIPO, 1, Rio Negro, AM, BR, 04/06/51, J. Falco, ex Alvarenga; 1, idem, 14/06/51, idem; 1 , idem, 15/04/51, idem; 1, idem, 16/06/51, idem; 1 , idem, 19/07/51, idem; 1 , idem, 22/06/51, idem; 1 , idem, 26/08/51, idem; 1 , idem, 28/06/51, idem; 1 , idem, 31/08/51, idem.

Stichotaenia crowsoni Bechyné, 1950, Revta Ent., Rio de J., 21: 115-156.

PARÁTIPO, 1, Rio Negrinho, SC, BR, 10/34, A. Maller, ex Mus. Nacional.

Syphraea geralis Bechyné, 1956, Ent. Arb., 7: 970-1071.

PARÁTIPO, 1, Campos do Jordão, SP, BR, 01/06, Luederwaldt, ex Alvarenga.

Syphraea iricolor Bechyné, 1956, Ent. Arb., 7: 970-1071.

PARÁTIPO, 1, S. Paulo (Ipiranga), SP, BR,29/04/07, Luederwaldt, ex Alvarenga.

Syphraea luederwaldti Bechyné, 1956, Ent. Arb., 7: 970-1071.

PARÁTIPO, 1, Campos do Jordão, SP, BR, 1906, Luederwaldt, ex Alvarenga.

Uroplata (Codiohispa) lantanae Buzzi, 1981, Revta bras. Ent., 25: 49-52.

HOLÓTIPO, Pato Branco, PR, BR, 08/10/79, J.A. Winder; PARÁTIPO, 10, idem, idem: 4, Campo Largo, PR, BR, 09/12/75, J.A. Winder; 7, Ponta Grossa (Vila Velha), PR, BR, 12/75, J.A. Winder.

Xenochalepus trilineatus utraque Uhmann, 1958, Iheringia, 9: 9.

PARÁTIPO, M, Nova Teutônia, SC, BR, 01/09/38, F. Plaumann, ex Plaumann.

\section{COCCINELLIDAE}

Argentipilosa martinsi Gordon \& Almeida, 1991, Revta bras. Ent., 35: 151-153. PARÁTIPO, 2, Itu (Faz.Pau d'Alho), SP, BR, 02/63, Werner \& Martins.

Argentipilosa nigra Gordon \& Almeida, 1991, Revta bras.Ent., 35: 151.

PARÁTIPO, 1, Rio de Janeiro (Corcovado), RJ, BR, 09/67, Alvarenga \& Seabra.

Mada amapa Gordon \& Almeida, 1986, Revta bras. Ent., 30: 418-419.

PARATIPO, 1, Serra do Navio, AP, BR, 04/10/57, J. Lane; 1, idem, 08/59, Bicelli.

Mada brasilia Gordon \& Almeida, 1986, Revta bras. Ent., 30: 367.

HOLÓTIPO, M, Jacaré (P.N.Xingu), MT, BR, 11/61, Alvarenga \& Werner;

PARÁTIPO, 2, Goiânia, GO, BR, 25/11/74, J. Moure; 1, Chapada dos Guimarães, MT, BR, 03/12/83, Exp. Dep. Zool.; 1, idem, 18/11/83, idem; 1, idem, 25/11/83, idem; 1, Jacaré (P.N. Xingu), MT, BR, 11/61, Alvarenga \& Werner; 5, Boa Esperança (Faz. Itaquerê), SP, BR, 25/01/64, K. Lenko;4, 
Tabatinga (Faz. Itaquerê), SP, BR, 01/12/63, K. Lenko.

Mada camargoi Gordon \& Almeida, 1986, Revta bras. Ent., 30: 371.

HOLÓTIPO, M, Óbidos, PA, BR, s/data, Balint; PARÁTIPO, 4, idem, idem.

Mada dentata Gordon \& Almeida, 1986, Revta bras. Ent., 30: 373.

HOLÓTIPO, M, S.Paulo de Olivença, AM, BR, 07/3, Wuccherpfenning; PARÁTIPO, 6, s/local., s/data.

Mada indistincta Gordon \& Almeida, 1986, Revta bras. Ent., 30: 367-368.

HOLÓTIPO, M, Rio de Janeiro (Corcovado), RJ, BR, 08/11/66, J. Moure.

Mada mourei Gordon \& Almeida, 1986, Revta bras. Ent., 30: 369.

HOLÓTIPO, M, Tijucas do Sul (Vossoroca), PR, BR, 15/01/72, Moure \& Aragão.

Mada pseudoapada Gordon \& Almeida, 1986, Revta bras. Ent., 30: 369.

HOLÓTIPO, M, Jacareacanga, PA, BR, 10/59, M. Alvarenga.

Neojauravia naeida Gordon \& Almeida, 1991, Revta bras. Ent., 35: 154.

HOLÓTIPO, M, Piracicaba, SP, BR, 12-19/03/87, F.D. Bennett;

ALÓTIPO, F, Piracicaba, SP, BR, 27/02/87, L. Crestana.

Psyllobora abancayana Almeida, 1992, Revta bras. Zool., 7: 421.

PARÁTIPO, 2, Abancay, AP, PE, 06/03/65, Ross \& Michelbacker.

Psyllobora amazonensis Almeida, 1992, Revta bras. Zool., 7: 411.

PARÁTIPO, F, Parintins, AM, BR, 01/40, Balint, ex Mus. Nacional.

Psyllobora balinti Almeida, 1992, Revta bras. Zool., 7: 411.

PARÁTIPO, 1, Cuiabá (Faz. Ricardo Franco), MT, BR, 11/03/61, J.\& B.

Bechyné, ex Mus. Nacional.

Psyllobora batesi Almeida, 1992, Revta bras. Zool., 7: 415-418.

PARÁTIPO, 9, s/local., AM, BR, 20/12/39, Balint; 20, Belterra, PA, BR, s/data, Balint.

Psyllobora borgmeiri Almeida, 1992, Revta bras. Zool., 7: 418.

PARÁTIPO, 1, Tucurrique, CR, s/data, Schild \& Burgdorf, ex Mus.

Nacional.

Psyllobora calida Almeida, 1985, Acta Biol. Par., Curitiba, 14: 74-76.

PARÁTIPO, 1, Rio de Janeiro (Corcovado), RJ, BR, 01/10/76, C.A.C. Seabra.

Psyllobora camargoi Almeida, 1985, Acta Biol. Par., Curitiba, 14: 71-73.

HOLÓTIPO, M, Nova Teutônia, SC, BR, 20/08/39, F. Plaumann;

PARÁTIPO, 2, idem, 04/50, idem; 1 , idem, 08/37, idem; 3, idem, 10/75, idem;

1, S. Paulo, SP, BR, 1939, F. Justus Jr.

Psyllobora confusa Almeida \& Marinoni, 1983, Revta bras. Ent., 27: 170-172.

HOLÓTIPO, Viçosa, MG, BR, 01/45, F. Camargo; PARÁTIPO, 5, idem, $1 / 45$, idem.

Psyllobora delicata Almeida, 1985, Acta Biol. Par.,Curitiba, 14: 65-68.

PARÁTIPO, 3, Salvador, BA, BR, 09/37, J.P.F; 1,Belém, PA, BR, 12/10/38, 
Hambl., \& Sauer.

Psyllobora equatoriana Almeida, 1992, Revta bras. Zool., 7: 423.

PARÁTIPO, 1, Cachimbiro, IM, EQ, 19/11/84, M. Garcia; 1, idem, 20/11/84, idem.

Psyllobora evanescens Almeida \& Marinoni, 1983, Revta bras. Ent., 27: 174-175.

PARÁTIPO, Esteio, RS, BR, 07/11/56, Pio Buck.

Psyllobora huancayensis Almeida, 1992, Revta bras. Zool., 7: 419-420.

PARÁTIPO, 20, Huancayo (Hotel Monterey), JU, PE, 11-23/01/67.

Psyllobora jalapaiensis Almeida, 1992, Revta bras. Zool., 7: 411-413.

PARATIPO, F, Turrialba, CA, CR, s/data, Schild \& Burgdorf, ex Mus. Nacional.

Psyllobora oxygnatha Almeida \& Marinoni, 1983, Revta bras. Ent., 27: 168-170.

PARÁTIPO, Vila Monte Verde, MG, BR, 10/12/69, J. Halik; 1, idem,

12/11/69, idem; 1, idem, 14/03/66, idem; 1, idem, 18/02/69, idem.

Psyllobora plaumanni Almeida, 1985, Acta Biol. Par., Curitiba, 14: 69-71.

HOLÓTIPO, M, Nova Teutônia, SC, BR, 11/39, F. Plaumann; PARÁTIPO,

1, idem, 02/08/37, idem; 1, idem, 08/08/37, idem; 10, idem, 10/62, idem; 2 , idem, 11/39, idem.

Psyllobora sosia Almeida \& Marinoni, 1983, Revta bras. Ent., 27: 172-174.

HOLÓTIPO, Araruama, RJ, BR, 25/08/45, F. Camargo; PARÁTIPO, 1 , Santa Mariana, PR, BR, 15/11/51, G.H. Nick; 3, Araruama, RJ, BR, 01/45, F. Camargo.

Psyllobora veraecrucis Almeida, 1992, Revta bras. Zool., 7: 413-414.

PARÁTIPO, 2, Xalapa, 25/10/66.

\section{CURCULIONIDAE}

Anthonomus acerolae Clark, 1988, Coleopts Bull., 42: 384-385.

HOLÓTIPO, M, Nova Soure, BA, BR, 1987, A.L. Mesquita; PARÁTIPO,

2 F, 3M, idem, idem; 1 F, 3M, Guarapari, ES, BR, 11/61, M. Alvarenga.

Anthonomus caceresensis Clark, 1988, Trans.Am. ent. Soc., 113: 346-348.

HOLÓTIPO, M, Cáceres, MT, BR, 19/10/61, F.M. Oliveira.

Anthonomus opous Clark, 1988, Trans. Am. ent. Soc., 113: 348-349.

PARÁTIPO, 1 F, 1 M, Ponta Grossa (Olaria), PR, BR, 11/46, F. Justus Jr.;

F, Ponta Grossa (Pedreira), PR, BR, 09/44, F. Justus Jr.; 3 M, Tijucas do Sul (Vossoroca), PR, BR, 15/01/72, Moure \& Aragao.

Anthonomus rhizonote Clark, 1988, Coleopts Bull., 42: 364-367.

PARÁTIPO, 5 F, 11 M, Batatais, SP, BR, 03/01/67, Pe. J. Moure; 1 F, 3 M, idem, 04/01/67, idem; M, Campo Grande, MS, BR, 09/10/61, F.M. Oliveira.

Aracanthus mourei Rosado-Neto, 1981, Revta bras. Ent., 25: 69-72.

HOLÓTIPO, M, Palmeira das Missões, RS, BR, 12/77, I. Dossa;

ALÓTIPO, F, idem, idem; PARÁTIPO, $2 \mathrm{M}$, idem, idem.

Argentinorhynchus bennetti O'Brien \& Wibmer, 1989, Ann. ent. Soc. Amer., 82: 
275-277.

PARÁTIPO, F, San Juan de Payara (5 km N), AP, VE, 12/77, O'Brien \& Wibmer; M, idem, 12/77, idem.

Atractomerus guaicuru Clark, 1989, Trans. Am. ent. Soc., 114: 337-338.

HOLÓTIPO, M, Sinop (BR-163, Km 500-600), MT, BR, 10/75, M. Alvarenga.

Atractomerus paiagua Clark, 1989, Trans. Am. ent. Soc., 114: 329-330.

HOLÓTIPO, M, Sinop (BR-163, Km 500-600), MT, BR, 10/74, M. Alvarenga.

Borgmeierus lineatus Bondar, 1943, Revta Ent., Rio de J., 14: 57-58.

CÓTIPO, 2 F, 1M, s/local., BA, BR, 03/43, Bondar ex Justus.

Dinomorphus cissophagus Bondar, 1948, Revta Ent., Rio de J., 19: 5-7.

CÓTIPO, M, Bom Jesus da Lapa, BA, BR, 10/47, Bondar, ex Bondar.

Eriocereophaga humeridens O'Brien, 1976, Coleopts Bull., 30: 305-307.

HOLÓTIPO, M, Arco Verde (próximo), PE, BR, 06/74, R.E. McFayden;

ALÓTIPO, F, idem, idem; PARÁTIPO, 2, M, idem, idem.

Erodiscus caruaru Vanin, 1986, Revta bras. Ent., 30: 475-476.

HOLÓTIPO, M, Caruaru, PE, BR, 04/72, M. Alvarenga.

Hadromeropsis (Hadromeropsis) excubitor Howden, 1982, Contr. Am. ent. Inst., 19: 78-80.

ALÓTIPO, F, Rio de Janeiro (Corcovado), RJ, BR, 08/65, M. Alvarenga \&

C. Seabra; PARÁTIPO, 2 F, 1 M, idem, 09/69, idem; F, idem, 10/60, idem;

F, idem, 18/09/61, Moure, Alvarenga \& Seabra.

Hadromeropsis (Hadromeropsis) plebeia Howden, 1982, Contr. Am. ent. Inst., 19: 62-65.

HOLÓTIPO, M, Olho d'Agua, PR, BR, 04/44, Rolando Linsing; ALÓTIPO, F, idem, idem; PARÁTIPO, F, Ortigueira, PR, BR, 01/44, F. Justus Jr.; F, Nova Teutônia, SC, BR, 02/76, F. Plaumann; F, idem, 03/77, idem; M, idem, 02/74, idem.

Hammatostylus erebus Vanin, 1986, Revta bras. Ent., 30: 629-631.

PARÁTIPO, M, Forte Príncipe da Beira, RO, BR, 07/11/61, F.M. Oliveira.

Hypnideus albomaculatus Rosado-Neto, 1986, An. Soc. ent. Brasil, 15: 141-146.

PARÁTIPO, F, Barro Colorado, ZC, PA, 28/06/78, H. Wolda.

Hypnideus bolivianus Rosado-Neto, 1988, An. Soc. ent. Brasil, 17: 484-486.

PARÁTIPO, F, Ichilo (Buena Vista), CO, BO, 11-12/48, L. Peña.

Loncophorus costalimai Clark, 1988, Quaest. ent., 24: 494-495.

HOLÓTIPO, M, Sinop (BR-163,Km 500-600), MT, BR, 10/74, M. Alvarenga .

Marshallius anacardii Almeida-Lima, 1979, An. Univ. Fed. Rural Pernamb., 4: 121-134.

HOLÓTIPO, M, Gravatá, PE, BR, 19/04/73, F. Lima \& V. Almeida; PARÁTIPO, 2F, 3 M, Mocajuba (Mangabeira), PA, BR, 10/53, O.M. Rego; 3 F, 1 M, Rio de Janeiro (Galeão), RJ, BR, 01/55, M.Alvarenga; F, idem, 
05/54, idem; M, s/local., BA, BR, 03/43, ex Justus; M, s/local., BA, BR, 06/43, ex Justus; 2 M, Mocajuba (Mangabeira), PA, BR, 02/53, O.M. Rego; 2 M, idem, 03/53, idem; $3 \mathrm{M}$, idem, 06/53, idem; $2 \mathrm{M}$, idem, 07/53, idem.

Marshallius bondari Rosado-Neto, 1989, Revta bras. Zool., 6: 517-520.

HOLÓTIPO, M, Nova Soure, BA, BR, 28/11/87, A.L.M. Mesquita;

PARÁTIPO, 3F, 7 M, idem, idem; 3 F, 1 M, idem, 01/88, S.W.P. Bispo.

Naupactus justus Bondar, 1949, Revta Ent., Rio de J., 20: 176-177.

CÓTIPO, F, Ponta Grossa, PR, BR, 02/45, F. Justus Jr., ex Bondar; M, Ponta

Grossa (Pedreira), PR, BR, 09/44, F. Justus Jr., ex Bondar.

Neohydronomus elegans O'Brien \& Wibmer, 1989, Coleopts Bull., 43:301-303.

PARÁTIPO, F, La Lima (12 mi NW), CO, HO, 23/07/74, C.\& L. O'Brien

\& Marshall, ex O'Brien; F, Acayucán (32 mi SW), VC, ME, 11/08/74, C. \&

L. O'Brien \& Marshall, ex O'Brien.

Ozoctenus vauriae Reichardt \& Vanin, 1973, Coleopts Bull., 27:87-90.

PARÁTIPO, 1 F, 1M, Linhares (Pq.Sooretama), ES, BR, 11/67, F.M.

Oliveira.

Pacholenus bifasciatus Vanin \& Reichardt, 1976, Papéis Avulsos Zool., 29: 166-167.

HOLÓTIPO, M, Rio Verde, MS, BR, 10/65, A. Maller.

Pacholenus hispidus Vanin \& Reichardt, 1976, Papéis Avulsos Zool., 29: 168-169.

PARÁTIPO, 1, Ponta Grossa (Lageado), PR, BR, 11/44, F. Justus Jr.

Parisoschoenus ananasi Moure, 1976, Dusenia, 9: 62-64.

HOLÓTIPO, M, Pitangueiras, SP, BR, 13/10/71, Eloys; PARÁTIPO, 10 F,

$4 \mathrm{M}$, idem, idem.

Philonis passiflorae O'Brien, 1984, SW. Ent., 9: 236.

HOLÓTIPO, M, Santo Amaro (Faz. Rocha), BA, BR, 26/11/78, P. Silva;

ALÓTIPO, F, idem, idem; PARÁTIPO, 7 F, 3 M, idem, idem; 3 F, 2 M, Rio

Real, BA, BR, 09/11/81, P. Silva.

Pistiacola fasciatus Wibmer \& O'Brien, 1989, SW.Ent., 14:400-402.

PARÁTIPO, 3 F, Cáceres, MT, BR, 05/08/84, D.P.Wojcik.

Pistiacoloides wojciki O'Brien, 1989, in SW. Ent., 14: 403-407.

PARÁTIPO, 3 F, 7 M, Cáceres, MT, BR, 05/08/84, D.P. Wojcik.

Plocetes falconiger Clark, 1982, Trans. Am. ent. Soc., 108: 48-49.

HOLÓTIPO, M, Curitiba, PR, BR, 16/02/66, C. Ext. D.Z.UFP; ALÓTIPO,

$\mathrm{F}$, idem, idem.

Plocetes senarius Clark, 1982, Trans.Am.ent.Soc., 108:64-65.

HOLÓTIPO, M, Goiânia, GO, BR, 25/11/74, J.S. Moure.

Polpones costalimai Bondar, 1943, Revta Ent., Rio de J., 14: 50.

CÓTIPO, F, s/local, BA, BR, 03/43, Bondar, ex Justus.

Rhinochenus mangabeirensis Whitehead, 1976, Quaest.ent., 12: 142-143.

HOLÓTIPO, F, Mocajuba (Mangabeira), PA, BR, 11/52, O. Rego, ex

Alvarenga. 
Rhinochenus pseudostigma Whitehead, 1976, Quaest. ent., 12: 155-156.

PARÁTIPO, M, Jacareacanga, PA, BR, 12/68, M.Alvarenga, ex Alvarenga.

Sibinia alvarenga Clark, 1978, Quaest. ent., 14: 166.

HOLÓTIPO, M, Pedra Azul, MG, BR, 11/72, M. Alvarenga; ALÓTIPO,

$F$, idem, idem.

Sibinia anfractoides Clark, 1978, Quaest. ent., 14: 288-291.

HOLÓTIPO, M, Pedra Azul, MG, BR, 11/72, M. Alvarenga.

Sibinia aspersoides Clark, 1978, Quaest. ent., 14: 302.

HOLÓTIPO, M, Ponta Grossa (Vila Velha), PR, BR, 19/01/69, J.S. Moure.

Sibinia earina Clark, 1978, Quaest. ent., 14: 297.

HOLÓTIPO, M, Pedra Azul, MG, BR, 11/72, M. Alvarenga; ALÓTIPO,

$\mathrm{F}$, idem, idem.

Sibinia fessa Clark, 1978, Quaest. ent., 14: 271-274.

HOLÓTIPO, M, Encruzilhada (Rod.Rio-Bahia,Km 965), BA, BR, 11/72,

Alvarenga \& Seabra; ALÓTIPO, idem, idem.

Sibinia gongropoda Clark, 1981 Coleopts Bull., 35: 227-228.

HOLOTIPO, M, S. José do Barreiro (Serra da Bocaina), SP, BR, 11/67,

Alvarenga \& Seabra; ALÓTIPO, F, idem, idem, 11/68, idem.

Sibinia hirritus Clark, 1978, Quaest. ent., 14: 248.

HOLÓTIPO, M, Encruzilhada (Rod. Rio-Bahia,Km 965), BA, BR, 11/72,

Alvarenga.

Sibinia impensa Clark, 1978, Quaest. ent., 14: 154-156.

HOLÓTIPO, M, Batatais, SP, BR, 02/01/68, J.S. Moure.

Sibinia ingenua Clark, 1978, Quaest.ent., 14:279.

HOLÓTIPO, M, Encruzilhada (Rod. Rio-Bahia,Km 965), BA, BR, 11/74,

M. Alvarenga; ALÓTIPO, F, idem, idem.

Sibinia nigripes Clark, 1978, Quaest. ent., 14: 177.

HOLÓTIPO, M, Pedra Azul, MG, BR, 11/72, M. Alvarenga; PARÁTIPO,

$F$, idem, idem.

Sibinia quinquemembrata Clark, 1978, Quaest. ent., 14: 267-268.

HOLÓTIPO, F, Balsa Nova (S. Luiz do Purunã), PR, BR, 20/01/68, Moure

\& Giacomel.

Sibinia turgidipes Clark, 1981, Coleopts Bull., 35: 225-227.

HOLÓTIPO, M, Alm. Tamandare (Tanguá), PR, BR, 16/01/79, Pedrosa;

ALÓTIPO, F, idem, idem; PARÁTIPO, $11 \mathrm{~F}, 8 \mathrm{M}$, idem, idem.

Sibinia warneri Clark, 1978, Quaest. ent., 14: 165.

HOLÓTIPO, M, Ponta Grossa (Vila Vilela), PR, BR, 12/55, F. Justus Jr.

Sicoderus bondari Vanin, 1986, Revta bras. Ent., 30: 539-542.

PARÁTIPO, F, Represa Rio Grande, RJ, BR, 09/01/67, F.M. Oliveira; M,

Linhares (Pq. Sooretama), ES, BR, 11/67, F.M. Oliveira.

Sicoderus tumidipectus Vanin, 1986, Revta bras. Ent., 30: 544-546.

HOLÓTIPO, M, Caruaru, PE, BR, 04/72, M. Alvarenga; PARÁTIPO, F, 
idem, idem.

Sternechus granulosus Rosado-Neto, 1977, Dusenia, 10: 140-142.

PARÁTIPO, F, Viçosa, MG, BR, 02/63, Werner, Martins \& Silva.

Sternechus mesosternalis Mendes, 1962, Arq. Mus. Nacional, Rio de J., 52: 133-134.

HOLÓTIPO, M, Goiatuba, GO, BR, 10/43; PARÁTIPO, F, idem, idem.

Sternechus triangulifer Rosado-Neto, 1977, Dusenia, 10: 125-127.

HOLÓTIPO, M, Barueri, SP, BR, 02/56, K. Lenko; ALÓTIPO, F, idem,

21/01/56, idem; PARÁTIPO, M, Felixlândia, MG, BR, 10/65, A. Maller; 2

M, Jaguariaiva, PR, BR, 01/47, M. Linsing.

Tyloderma affine Wibmer, 1989, Evolutionary Monographs, 11: 50-52.

PARÁTIPO, M, Clorinda (34 km SW), FO, AR, 26/01/89, C.\& L. O’Brien

\& G. Wibmer.

Tyloderma brevisquameum Wibmer, 1989, Evolutionary Monographs, 11: 73-74.

PARÁTIPO, 7 F, Poconé (17 Km S), MT, BR, 24/04/81, D.P. Wojcik; 3 M, idem, idem.

Tyloderma elongatum Wibmer, 1989, Evolutionary Monographs, 11: 92-94.

PARÁTIPO, 1 F, 1 M, San Pedro, LC, UR, 06/01/79, G.J. \& Z. Wibmer.

Tyloderma glabrescens Wibmer, 1989, Evolutionary Monographs, 11: 46-47.

PARÁTIPO, F, Puerto Banegas (10 mi W), SC, BO, 25/03/78, G.B.

Marshall.

Tyloderma hustachei Wibmer, 1989, Evolutionary Monographs, 11: 26-29.

PARÁTIPO, 4 F, 4 M, Jacareacanga, PA, BR, 12/68, M. Alvarenga; 2 F, S.

Carlos, SP, BR, 01/10/82, M. Hoffmann; F, idem, 12/10/82, Moure \&

Marinoni; F, idem, 25/10/82, M. Hoffmann; 1 F, 1 M, 28/10/82, M. Hoffmann;

M, Sete Lagoas (IPEACO), MG, BR, 20/10/69, Becker.

Tyloderma lacordairei Wibmer, 1989, Evolutionary Monographs, 11: 45-46.

PARÁTIPO, 1F, 1 M, Lules (5 Km S), TU, AR, 19/10/68, C. \& L. O'Brien.

Tyloderma natator Wibmer, 1989, Evolutionary Monographs, 11: 48-49.

PARÁTIPO, 2 F, 2 M, Reducto, LC, UR, 29/12/78, G.J.\& Z.Wibmer.

Tyloderma obrieni Wibmer, 1989, Evolutionary Monographs, 11: 91-92.

PARÁTIPO, M, Zarate (13 mi E), BA, AR, 14/04/78, C.W. O'Brien.

Tyloderma sayi Wibmer, 1989, Evolutionary Monographs, 11: 60-61.

PARÁTIPO, 1 F, 1 M, Calabozo (13 Km SW), GU, VE, 26/07/88, O'Brien,

O'Brien \& Wibmer.

Tyloderma simile Wibmer, 1989, Evolutionary Monographs, 11: 66-67.

PARÁTIPO, M, Arinos, MG, BR, 06-08/11/64, Exp. Dep. Zool.

\section{EROTYLIDAE}

Brachysphaenus conformis minor Guerin, 1956, Dusenia, 7: 50.

PARÁTIPO, 1, Borba (Mata de Borba), AM, BR, 04/43, A. Parko, ex Alvarenga; 2, Mocajuba (Mangabeira), PA, BR, 06/53, Orlando Rego, ex 
Alvarenga.

Erotylus baylei Alvarenga, 1970, Atas Soc. Biol. Rio de J., 14: 149-150.

HOLÓTIPO, Óbidos, AM, BR, 10/31, B. Pohl, ex Alvarenga.

Erotylus pretiosus geometricus Guerin, 1956, Dusenia, 7: 58.

PARÁTIPO, 1, s/local., AM, BR, s/data, A. Parko, ex Alvarenga.

Eurycardius seabraia Alvarenga, 1970, Atas Soc. Biol. Rio de J., 14: 117-118.

HOLÓTIPO, Córrego do Itá, ES, BR, 11/66, W. Zikán, ex Alvarenga.

Priotelus quadrimaculatus Guerin, 1956, Dusenia, 7: 60.

TIPO, 1, Mocajuba (Mangabeira), PA, BR, 05/53, Orlando Rego, ex Alvarenga.

Pselaphacus sparsus paraensis Guerin, 1956, Dusenia, 7: 61.

PARÁTIPO, 6, Mocajuba (Mangabeira), PA, BR, 06/53, Orlando Rego, ex Alvarenga.

\section{GYRINIDAE}

Gyretes angusticinctus Ochs, 1957, Senckenberg., biol., 38: 214.

HOLÓTIPO, F, Nova Teutônia, SC, BR, 12/61, F. Plaumann, ex Plaumann;

PARÁTIPO, F, idem, idem.

Gyretes assimilis Ochs, 1953, Opusc. ent., 18: 141.

HOLÓTIPO, F, Marília, SP, BR, 05/47, F. Plaumann, ex Plaumann.

Gyretes bruchi nova-teutoniae Ochs, 1960, Senckenberg., biol., 41: 181.

PARÁTIPO, 40, Lagoa Vermelha (R.S.Rita) $\left(28^{\circ} 20^{\prime} \mathrm{S}, 51^{\circ} 18^{\prime} \mathrm{W}\right)$, RS, BR,

04/69, F. Plaumann, ex Plaumann.

Gyretes clipealis Ochs, 1964, Ent. Mitt. zool. StInst. zool. Mus. Hamb., 3: 1.

PARÁTIPO, 2, Guarapuava (Rio Coitinho) 1100m, PR, BR, 03/63, F.

Plaumann, ex Plaumann.

Gyretes connatus Ochs, 1960, Senckenberg., biol., 41: 187.

PARÁTIPO, 4, Mariópolis(Arroio Lambedor), PR, BR,01/58, F.

Plaumann, ex Plaumann.

Gyretes elegans Ochs, 1960, Senckenberg., biol., 41: 186.

HOLOTIPO, Nova Teutônia, SC, BR, 04/58, F. Plaumann, ex Plaumann.

Gyretes fraudulentus neuter Ochs, 1960, Senckenberg., biol., 41: 182.

PARÁTIPO, 5, Linha Facão, SC, BR, 01/57, F. Plaumann ex Plaumann.

Gyretes gibbosus Ochs, 1953, Opusc. ent., 18: 146.

HOLÓTIPO, M, Jaborá, SC, BR, 10/47, F. Plaumann ex Plaumann.

Gyretes laevis australis Ochs, 1960, Senckenberg., biol., 41: 311.

PARÁTIPO, 30, Osório (Lagoa dos Barros)(2953'S,50²0’W), RS, BR, 04/59, F. Plaumann, ex Plaumann.

Gyretes latipes Ochs, 1960, Senckenberg., biol., 41: 182.

HOLOTIPO, Nova Teutônia, SC, BR, 12/56, F. Plaumann, ex Plaumann;

PARÁTIPO, idem, idem.

Gyretes minusculus Ochs, 1953, Opusc. ent., 18: 143-144. 
HOLÓTIPO, F, Nova Teutônia, SC, BR, 16/02/44, F. Plaumann, ex Plaumann.

Gyretes oblongus iridipennis Ochs, 1960, Senckenberg., biol., 41: 312.

PARÁTIPO, 1, Osório (Lagoa dos Barros)( $\left.29^{\circ} 53^{\prime} \mathrm{S}, 50^{\circ} 20^{\prime} \mathrm{W}\right)$, RS, BR,

04/59, F. Plaumann, ex Plaumann; 2, Torres (Lagoa Itapeva)

$\left(29^{\circ} 28^{\prime}\right.$ S, $\left.50^{\circ} 00^{\prime} W^{\prime}\right)$, RS, BR, 04/59, F. Plaumann, ex Plaumann.

Gyretes pauxillus Ochs, 1964, Ent. Mitt. zool. StInst. zool. Mus. Hamb.,3: 4.

PARÁTIPO, 2, Guarapuava (R. Camp. Novo) $\left(25^{\circ} 16^{\prime}\right.$ S, $\left.51^{\circ} 29^{\prime} \mathrm{W}\right), \mathrm{PR}, \mathrm{BR}$,

03/63, F. Plaumann, ex Plaumann.

Gyretes scitus Ochs, 1960, Senckenberg., biol., 41: 309.

HOLÓTIPO, F, Jaquirana (Rio Tainhas) (28 $55^{\circ}$ 'S, $\left.50^{\circ} 35 W^{\prime}\right)$, RS, BR, 04/59,

F. Plaumann, ex Plaumann.

Gyretes tarsalis Ochs, 1953, Opusc. ent., 18: 147-148.

HOLÓTIPO, M, Jaborá, SC, BR, 10/47, F. Plaumann, ex Plaumann.

Gyretes tarsalis chapadensis Ochs, 1960, Senckenberg., biol., 41: 310.

PARÁTIPO, 15 , Chapada (Arroio do Barreiro) $\left(28^{\circ} 48^{\prime} \mathrm{S}, 50^{\circ} 26^{\prime} \mathrm{W}\right), \mathrm{RS}, \mathrm{BR}$,

04/59, F. Plaumann, ex Plaumann.

Gyretes torrenticola alticola Ochs, 1964, Ent. Mitt. zool. StInst. zool. Mus.

Hamb.,3: 1 .

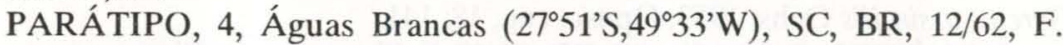
Plaumann, ex Plaumann.

\section{SCARABAEIDAE}

Agacephala margaridae Alvarenga, 1958, Revta bras. Biol., 18: 47-54.

PARÁTIPO, Cachimbo, PA, BR, 25/09-10/58, Travassos, Oliveira \& Adão, ex Alvarenga.

\section{SCOLYTIDAE.}

Amphicranus rasilis Schedl, 1950, Dusenia, 1: 174-175.

TIPO, 4 F, 4 M, Nova Teutônia, SC, BR, 08/44, F. Plaumann, ex Plaumann.

Amphicranus truncatorum Schedl, 1950, Dusenia, 1: 175-176.

TIPO, 4 F, 3 M, Nova Teutônia, SC, BR, 04/41, F. Plaumann, ex Plaumann.

Brachydendrulus minutissimus Schedl, 1954 Dusenia, 5: 35.

TIPO, 1, Rondon $\left(24^{\circ} 38^{\prime} \mathrm{S}, 54^{\circ} 07^{\prime} \mathrm{W}\right)$, PR, BR, 10/52, F. Plaumann, ex Plaumann.

Brachydendrulus paranae Schedl, 1954. Dusenia, 5: 34.

TIPO, 1, Rondon ( $\left.24^{\circ} 38^{\prime} \mathrm{S}, 54^{\circ} 07^{\prime} \mathrm{W}\right)$, PR, BR, 10/52, F. Plaumann, ex Plaumann.

Brachyspartus bisetosus Schedl, 1954, Dusenia, 5: 38.


Plaumann, ex Plaumann.

Breviophthorus sulcatus Schedl, 1959, Beitr. Ent., 9: 552. 
PARÁTIPO, F, Rio Caraguatá ( $\left.21^{\circ} 48^{\prime} \mathrm{S} 52^{\circ} 27^{\prime} \mathrm{W}\right)$, MS, BR, 09/53, F. Plaumann, ex Plaumann; 2 F, idem, 06/53, idem; M, idem, 1953, idem.

Ceratolepis barbatus Schedl, 1954, Dusenia, 5: 24-25.

TIPO, 1, Nova Teutônia, SC, BR, 08/39, F. Plaumann, ex Plaumann.

Chramesus aberrans Schedl, 1951, Dusenia, 2: 90.

TIPO, 4, Nova Teutônia, SC, BR, 08/41, F. Plaumann, ex Plaumann.

Chramesus setiger Schedl, 1951, Dusenia, 2: 92.

PARÁTIPO, 6, Nova Teutônia, SC, BR, 08/46, F. Plaumann, ex Plaumann.

Cnemonyx creber Schedl, 1951, Dusenia, 2: 76-77.

TIPO, 4, Nova Teutônia, SC, BR, 07/44, F. Plaumann, ex Plaumann.

Cnemonyx minor Schedl, 1951, Dusenia, 2: 75-76.

TIPO, 4, Nova Teutônia, SC, BR, 07/44, F. Plaumann, ex Plaumann.

Cnesinus dryographus Schedl, 1951, Dusenia, 2: 78-79.

TIPO, 2, Nova Teutônia, SC, BR, 08/44, F. Plaumann, ex Plaumann.

Cnesinus laevicollis Schedl, 1951, Dusenia, 2: 79-80.

TIPO, 4, Nova Teutônia, SC, BR, 05/44, F.Plaumann, ex Plaumann.

Cnesinus nova-teutonicus Schedl, 1951, Dusenia, 2: 77-78.

TIPO, 1, Nova Teutônia, SC, BR, 02/37, F. Plaumann, ex Plaumann.

Cnesinus schoenherri Schedl, 1976, Entoml. Abhandl. Mus. Tierk., Dresden, 41:63.

TIPO, 1, Rio Negrinho, SC, BR, 03/72, J. Schoenherr.

Corthycyclon ustum Schedl, 1951, Dusenia, 2: 128.

TIPO, 1, Nova Teutônia, SC, BR, 03/41, F. Plaumann, ex Plaumann.

Corthylus nudipennis Schedl, 1950, Dusenia, 1: 155-156.

TIPO, 2 M, Nova Teutônia, SC, BR, 08/41, F.Plaumann, ex Plaumann.

Cryptocleptes abbreviatus Schedl, 1954, Dusenia, 5: 26-27.

TIPO, 4 F, 4 M, Rondon ( $24^{\circ} 38^{\prime}$ S $\left.54^{\circ} 07^{\prime} \mathrm{W}\right)$, PR, BR, 10/52, F. Plaumann, ex

Plaumann.

Cryptocleptes bellus Schedl, 1954, Dusenia, 5: 28-29.

TIPO, 1 F, 1 M, Rondon ( $\left.24^{\circ} 38^{\prime}, 54^{\circ} 07^{\prime}\right)$, PR, BR, 09/52, F. Plaumann, ex Plaumann.

Cryptocleptes dimorphus Schedl, 1954, Dusenia, 5: 25-26.

TIPO, 5 F, 5 M, Rio Caraguatá ( $\left.21^{\circ} 48^{\prime} \mathrm{S}, 52^{\circ} 27^{\prime} \mathrm{W}\right)$, MS, BR, 03/53, F.

Plaumann, ex Plaumann.

Cryptocleptes plaumanni Schedl, 1951, Dusenia, 2: 106-107.

TIPO, 4 F, 4 M, Nova Teutônia, SC, BR, 09/44, F. Plaumann, ex Plaumann.

Gnathocranus nova-teutonicus Schedl, 1951, Dusenia, 2: 116.

TIPO, F, Nova Teutônia, SC, BR, 01/41, F. Plaumann, ex Plaumann.

Hexacolus glabratus Schedl, 1954, Dusenia, 5: 23-24.

PARATIPO, 2 F, Nova Teutônia, SC, BR, 17/11/49, F. Plaumann, ex

Plaumann; 2 F, idem, 19/11/49, idem; TIPO, 2 M, idem, 08/42, idem.

Hexacolus glabrellus Schedl, 1954, Dusenia, 5: 21-22. 
TIPO, 5 F, 4 M, Rondon ( $24^{\circ} 38^{\prime}$ S, $\left.54^{\circ} 07 W^{\prime}\right)$, PR, BR, 08/52, F. Plaumann, ex Plaumann.

Hylocurus bidentatus Schedl, 1950, Dusenia, 1: 149-150.

TIPO, 4, 2 F, 2 M, Nova Teutônia, SC, BR, 04/41, F. Plaumann, ex Plaumann. Hylocurus dubius Schedl, 1959, Beitr. Ent., 9: 547.

PARÁTIPO, $3 \mathrm{~F}, 2 \mathrm{M}$, Rondon ( $\left.24^{\circ} 38^{\prime} \mathrm{S}, 54^{\circ} 07^{\prime} \mathrm{W}\right)$, PR, BR, 11/52, F. Plaumann, ex Plaumann.

Hylocurus interruptus Schedl, 1959, Beitr. Ent., 9: 548.

PARÁTIPO, 10, $1 \mathrm{M}$, Nova Teutônia, SC, BR, 12/40, F. Plaumann, ex Plaumann.

Hylocurus obscurus Schedl, 1949, Revta Ent., Rio de J., 6: 390.

PARÁTIPO, 5, $1 \mathrm{M}$, Nova Teutônia, SC, BR, 08/41, F. Plaumann, ex Plaumann.

Hylocurus pilosus Schedl, 1950, Dusenia, 1: 151.

TIPO, 1, Nova Teutônia, SC,BR, 07/41, F. Plaumann, ex Plaumann, 1 F, idem, 08/41, idem.

Hylocurus pseudoimpar Schedl, 1954, Dusenia, 5: 30-31.

TIPO, 7, 1 M, Nova Teutônia, SC, BR, 10/41, F. Plaumann, ex Plaumann.

Hylocurus subgranulatus Schedl, 1954, Dusenia, 5: 31-33.

TIPO, 6, 1 F, 1 M, Rio Caraguatá (21 $48^{\circ}$ ' $\left.52^{\circ} 27^{\prime} \mathrm{W}\right)$, MS, BR, 03/53, F. Plaumann, ex Plaumann.

Hypothenemus eximius Schedl, 1951, Dusenia, 2: 99-100.

TIPO, 3, Nova Teutônia, SC, BR, 09/44, F. Plaumann, ex Plaumann.

Hypothenemus parilis Schedl, 1951, Dusenia, 2: 100-101.

TIPO, 3, Nova Teutônia, SC, BR, 11/42, F.Plaumann, ex Plaumann.

Loganius difformis Schedl, 1951, Dusenia, 2: 74-75.

TIPO, 4, Nova Teutônia, SC, BR, 07/41, F. Plaumann, ex Plaumann.

Metacorthylus niger Schedl, 1954, Dusenia, 5: 43-44.

TIPO, 2, Nova Teutônia, SC, BR, 09/10/49, F. Plaumann, ex Plaumann.

Micracis giganteus Schedl, 1950, Dusenia, 1: 152-153.

TIPO, 3 F, 3 M, Nova Teutônia, SC, BR, 04/41, F. Plaumann, ex Plaumann.

Microcorthylus minimus Schedl, 1950, Dusenia, 1: 160.

TIPO, 6 F, Nova Teutônia, SC, BR, 04/44, F. Plaumann, ex Plaumann.

Neodryocoetes grandis Schedl, 1954, Dusenia, 5: 35-36.

TIPO, 1 F, 3 M, Rio Caraguatá ( $21^{\circ} 48^{\prime}$ S, $\left.52^{\circ} 27^{\prime} \mathrm{W}\right)$, MS, BR, 04/53, F.

Plaumann, ex Plaumann.

Neomips brasiliensis Schedl, 1954, Dusenia, 5: 38.

PARÁTIPO, $1 \mathrm{~F}, 3 \mathrm{M}$, Rio Caraguatá ( $\left.21^{\circ} 48^{\prime} \mathrm{S}, 52^{\circ} 27^{\prime} \mathrm{W}\right)$, MS, BR, 04/53, F. Plaumann, ex Plaumann; M, idem, 03/53, idem; TIPO, 2F, idem, 05/53, idem.

Phloeotribus cylindricus Schedl, 1951, Dusenia, 2: 82-83.

PARÁTIPO, 1 F, 1 M, Nova Teutônia, SC, BR, 11/40, F. Plaumann, ex Plaumann; TIPO, 2 F, 3 M, idem, idem. 
Phloeotribus erosus Schedl, 1951, Dusenia, 2: 83-84.

TIPO, 4, Nova Teutônia, SC, BR, 06/44, F. Plaumann, ex Plaumann.

Phrixosoma brasiliensis Schedl, 1959, Beitr. Ent., 9: 546.

PARÁTIPO, 2, Rio Caraguatá ( $21^{\circ} 48^{\prime}$ 'S, $\left.52^{\circ} 27^{\prime} \mathrm{W}\right)$, MS, BR, 15/10/53, F. Plaumann, ex Plaumann.

Phthorophloeus nova-teutonicus Schedl, 1951, Dusenia, 2: 84-85.

TIPO, 4, Nova Teutônia, SC, BR, 08/41, F. Plaumann, ex Plaumann.

Pityophthorus mandibularis Schedl, 1951, Dusenia, 2: 113-114.

TIPO, 3 F, 4 M, Nova Teutônia, SC, BR, 11/46, F. Plaumann, ex Plaumann.

Pseudochramesus abbreviatus Schedl, 1951, Dusenia, 2: 94.

TIPO, M, Nova Teutônia, SC, BR, 08/46, F. Plaumann, ex Plaumann.

Pseudochramesus brasiliensis Schedl, 1949, Revta bras. Biol., 9: 265.

TIPO, 3, Nova Teutônia, SC, BR, 10/44, F. Plaumann, ex Plaumann.

Pseudochramesus opacus Schedl, 1963, Reichenbachia, 1: 216.

TIPO, 5 M, Rio Caraguatá ( $\left.21^{\circ} 48^{\prime} \mathrm{S}, 52^{\circ} 27^{\prime} \mathrm{W}\right)$, MS, BR, 03/53, F. Plaumann, ex Plaumann.

Pseudothysanoes unimodus Schedl, 1959, Beitr. Ent., 9: 549.

PARÁTIPO, 8, Rio Caraguatá $\left(21^{\circ} 48^{\prime} \mathrm{S}, 52^{\circ} 27^{\prime} \mathrm{W}\right), \mathrm{MS}, \mathrm{BR}, 03 / 53$, F.

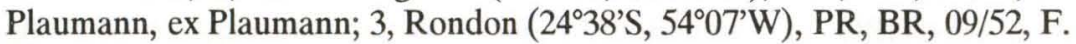
Plaumann, ex Plaumann.

Pterocyclon bicallosum Schedl, 1939, Mitt. Münch. ent. Ges., 29: 577.

TIPO, 2, Nova Teutônia, SC, BR, 08/39, F. Plaumann, ex Plaumann.

Pterocyclon gracilior Schedl, 1959, Beitr. Ent., 9: 553.

PARÁTIPO, 4 F, 4 M, Nova Teutônia, SC, BR, 04/44, F. Plaumann, ex Plaumann.

Pterocyclon minutissimum Schedl, 1954. Dusenia, 5: 40-41.

PARÁTIPO, 2 F, 2 M, Rio Caraguatá ( $\left.21^{\circ} 48^{\prime} \mathrm{S} 52^{\circ} 27^{\prime} \mathrm{W}\right), \mathrm{MS}, \mathrm{BR}, 05 / 53$, F.

Plaumann, ex Plaumann.

Pterocyclon minutum Schedl, 1939, Mitt. Münch. ent. Ges., 29: 577.

TIPO, 6, Nova Teutônia, SC, BR, 08/37, F. Plaumann, ex Plaumann; 2, idem, $08 / 39$, idem.

Pterocyclon plaumanni Schedl, 1937, Arb. morph. taxon. Ent. Berl., 4: 68.

TIPO, 4 F, 4 M, Nova Teutônia, SC, BR, 193?, F. Plaumann, ex Plaumann.

Pterocyclon semipallens Schedl, 1954, Dusenia, 5: 41-42.

TIPO, 2 M, Rio Caraguatá ( $21^{\circ} 48^{\prime}$ S $\left.52^{\circ} 27^{\prime} \mathrm{W}\right)$, MS, BR, 03/53, F. Plaumann, ex Plaumann.

Ptilopodius nova-teutonicus Schedl, 1951, Dusenia, 2: 105.

TIPO, 1, Nova Teutônia, SC, BR, 09/46, F. Plaumann, ex Plaumann.

Scolytus nova-teutonicus Schedl, 1937, Arch.I nst. Biol. Veg., 3: 162.

TIPO, 2, Nova Teutônia, SC, BR, 193?., F. Plaumann, ex Plaumann.

Tricolus gracilipennis Schedl, 1950, Dusenia, 1: 170-171.

PARÁTIPO, 3, Nova Teutônia, SC, BR, 08/41, F. Plaumann, ex Plaumann. 
Tricolus plaumanni Schedl, 1954, Dusenia, 5: 40.

TIPO, 3, Rondon ( $24^{\circ} 38^{\prime}$ S, $\left.54^{\circ} 07^{\prime} \mathrm{W}\right)$, PR, BR, 09/52, F. Plaumann, ex Plaumann.

Tricolus senex Schedl, 1939, Mitt. Münch. ent. Ges., 29: 580.

TIPO, 4, Nova Teutônia, SC, BR, 08/37, F. Plaumann, ex Plaumann.

Tricolus spheniscus Schedl, 1939, Mitt. Münch. ent. Ges., 29: 581.

TIPO, 5 F, Nova Teutônia, SC, BR, 07/37, F. Plaumann, ex Plaumann.

Xyleborus nova-teutonicus Schedl, 1954, Dusenia, 5: 47-48.

TIPO, 3, Nova Teutônia, SC, BR, 02/50, F. Plaumann, ex Plaumann.

Xyleborus obtusotruncatus Schedl, 1949, Revta bras. Biol., 9: 271.

TIPO, 6, Nova Teutônia, SC, BR, 12/44, F. Plaumann, ex Plaumann.

Xyleborus scaber Schedl, 1949, Revta bras. Biol., 9: 273.

PARÁTIPO, M, Nova Teutônia, SC, BR, 07/37, F. Plaumann, ex Plaumann; TIPO, 1 , idem, 05/35, idem.

\section{STAPHYLINIDAE}

Tetradonia alvarengai Borgmeier, 1959, Bol. Mus. Nac., N. S., Zool., Rio de J., 214: 11.

PARÁTIPO, 3 F, Oiapoque, AP, BR, 05/59, M.Alvarenga, ex Alvarenga.

Tetradonia amapaensis Borgmeier, 1959, Bol. Mus. Nac., N. S., Zool., Rio de J., 214: 12.

PARÁTIPO, F, Oiapoque, AP, BR, 05/59, M. Alvarenga, ex Alvarenga.

Tetradonia longicornis Borgmeier, 1959, Bol. Mus. Nac., N. S., Zool., Rio de J., 214: 10.

PARÁTIPO, 3 F, Oiapoque, AP, BR, 05/59, M. Alvarenga, ex Alvarenga.

AGRADECIMENTOS. Agradecemos a colaboração de José Henrique Pedrosa-Macedo, Zundir José Buzzi e Cibele S.Ribeiro-Costa, pelas informações prestadas sobre Scolytidae, Chrysomelidae (Cassidinae) e Bruchidae.

Recebido em 02XI.1992; aceito em 06.VI.1993. 